\title{
Revisiting Jiang's dynamic continuum model for urban cities
}

Jie $\mathrm{Du}^{1}$, S.C. Wong ${ }^{2}$, Chi-Wang Shu ${ }^{3}$, Tao Xiong ${ }^{4}$, Mengping Zhang ${ }^{5}$, and Keechoo Choi ${ }^{6}$

Abstract: Jiang et al. (Transportation Research Part B, 2011, 45(2), 343-363) proposed a predictive continuum dynamic user-optimal (PDUO-C) model to investigate the dynamic characteristics of traffic flow and the corresponding route-choice behavior of travelers. Their modeled region is a dense urban city that is arbitrary in shape and has a single central business district (CBD). However, we argue that the model is not well posed due to an inconsistency in the route-choice strategy under certain conditions. To overcome this inconsistency, we revisit the PDUO-C problem, and construct an improved path-choice strategy. The improved model consists of a conservation law to govern the density, in which the flow direction is determined by the improved path-choice strategy, and a Hamilton-Jacobi equation to compute the total travel cost. The simultaneous satisfaction of both equations can be treated as a fixed-point problem. A self-adaptive method of successive averages (MSA) is proposed to solve this fixed-point problem. This method can automatically determine the optimal MSA step size using the least squares approach. Numerical examples are used to demonstrate the effectiveness of the model and the solution algorithm.

Key Words: Continuum model, Dynamic traffic assignment, Predictive user equilibrium, Path-choice strategy, Conservation law, Hamilton-Jacobi equation, Fixed-point problem, Method of successive averages (MSA), Self-adaptive.

\footnotetext{
${ }^{1}$ Department of Mathematics, University of Science and Technology of China, Hefei, Anhui, P.R. China. E-mail: dujie@mail.ustc.edu.cn

${ }^{2}$ Department of Civil Engineering, The University of Hong Kong, Pokfulam Road, Hong Kong, P.R. China. E-mail: hhecwsc@hkucc.hku.hk

${ }^{3}$ Division of Applied Mathematics, Brown University, Providence, RI, USA. E-mail: shu@dam.brown.edu

${ }^{4}$ Department of Mathematics, University of Houston, Houston, TX, USA. E-mail: txiong@math.uh.edu

${ }^{5}$ Department of Mathematics, University of Science and Technology of China, Hefei, Anhui, P.R. China. E-mail: mpzhang@ustc.edu.cn

${ }^{6}$ Department of Transportation Engineering, TOD-based Sustainable Urban Transportation Center, Ajou University, Korea. E-mail: keechoo@ajou.ac.kr
} 


\section{Introduction}

The traffic equilibrium problem has received much attention in recent decades. The large number of studies devoted to the problem can be generally classified into two categories: those that have used either discrete or continuum modeling approaches. The discrete modeling approach is the conventional methodology used for detailed studies of travel patterns in road network systems, in which each road link within the network is modeled separately (Lee, 2002). In contrast, the continuum modeling approach takes the modeling region as a continuum and focuses on the overall behavior of travelers at the macroscopic level. In the continuum modeling approach, the differences between adjacent areas within a network are assumed to be relatively small compared with the variation over the entire area, and hence the characteristics of the network, such as the flow intensity, density and travel cost, can be represented by smooth mathematical functions (Vaughan, 1987).

The majority of the studies that have used the continuum modeling approach for urban cities have been confined to static cases. Studies on the static continuum modeling of traffic equilibrium problems can be broadly divided into two categories: those that have focused on either specific or arbitrary city configurations. The specific city configuration study area is approximated by an idealized domain for analysis. A circular region specification has most commonly been adopted. For example, Blumenfeld and Weiss (1970), Lam and Newell (1967), Williams and Ortuzar (1976) and Zitron (1978) assumed the modeling region to be circular. Other specifications such as linear (Tong and Wong, 1997; Vaughan, 1985; Zhang et al., 2008), bilinear (Buckley, 1979) and rectangular (Dafermos, 1980) specifications have also been considered for various kinds of trip assignment and distribution problems. In contrast, a continuum model approach with an arbitrary city configuration can be used for regions of any arbitrary shape, and does not require paths within the region to run in a predefined travel direction (Blumenfeld, 1977; Taguchi and Iri, 1982; Wong et al., 1998). Extensions of the continuum modeling 
approach have also been proposed for multiple destinations and/or multiple user classes (Wong, 1998; Ho et al., 2006, 2013; Yin et al., 2013).

As the temporal variation of flow and cost are not considered in the static models, they cannot be used to examine elements such as travelers' departure/arrival time choices or dynamic traffic management and control. Dynamic traffic assignment (DTA) has received much attention when addressing these problems in recent decades. An important component of DTA is the travel choice problem, which models travel behavior. Three major problems are considered in this component: the dynamic system-optimal (DSO) problem, which aims to minimize the total travel cost of the system (Chow, 2009; Lo and Szeto, 2005); the reactive dynamic user-optimal (RDUO) problem, in which travelers choose their route using instantaneous information and change their choice in a reactive manner (Boyce et al., 1993; Kuwahara and Akamatsu, 2001); and the predictive dynamic user-optimal (PDUO) problem, in which travelers are assumed to have perfect information about the modeled domain they can use to choose the route that minimizes the actual travel cost (Hoogendoorn and Bovy, 2004; Lo and Szeto, 2002; Szeto and Lo, 2004; Tong and Wong, 2000).

However, most of the continuum models for urban cities rely on the static assumption of the demand and supply sides of the transportation system. To improve the capacity of the continuum model and govern its dynamic changes and practicality in large-scale/dense networks, Jiang et al. (2011) discussed the formulation of and proposed a solution algorithm for a predictive continuum dynamic user-optimal (PDUO-C) problem for a large, dense urban city with a single central business district (CBD). Travelers are continuously distributed across the city and travel to the CBD within the modeling region for a given time-dependent travel demand. The proposed model consists of a flow conservation equation to compute the density, and a time-dependent HamiltonJacobi equation and Eikonal equation to compute the travel cost. In this paper, we show that one of the equations used to compute the travel cost is mathematically un- 
necessary. However, the user-optimal conditions in Jiang et al. (2011) required both of these equations to be satisfied. The main problem with this inconsistency comes from the requirements in the path-choice strategy, illustrated as follows.

To overcome this inconsistency, we revisit the PDUO-C problem and propose an improved path-choice strategy. The improved model consists of two PDEs: a conservation law, which governs the density in the city using the improved path-choice strategy, and a time-dependent Hamilton-Jacobi equation to compute the total travel cost, which differs from the equation in Jiang et al. (2011). To solve our model numerically, we adopt the Lax-Friedrichs scheme, which is a conservative monotone scheme with properties such as the maximum principle and total variation diminishing (TVD). Unlike other problems in which the initial conditions of both equations are set at the beginning of the modeling period, the initial conditions of these two equations in our model are set differently, with one at the beginning and the other at the end of the modeling period. Therefore, we cannot solve them together as usual. In fact, this model can be treated as a fixed-point problem, which can be solved by iteration methods such as the method of successive averages (MSA) (Bar-Gera and Boyce, 2006; Liu et al., 2009; Nagurney and Zhang, 1996; Polyak, 1990; Robbins and Monro, 1951). Among the various types of MSA, the MSA with a constant step size (Bar-Gera and Boyce, 2006) is very efficient. However, this method needs to estimate the optimal step size according to past experience and decrease it as necessary. Based on a formula proposed by Bar-Gera and Boyce (2006), we construct a self-adaptive MSA that can automatically determine the optimal step size using the least squares method without prior information. The numerical results show that this new constructed self-adaptive MSA is much faster than the conventional methods.

The remainder of this paper is organized as follows. The description of the PDUO$\mathrm{C}$ problem is given in the next section. In Section 3, we review the formulation of the original PDUO-C model in Jiang et al. (2011) and point out the inconsistency. In Section 
4, we discuss the formulation of an improved model. We describe the solution algorithm in Section 5. In Section 6, we present the numerical results and compare them with the MSAs for other step sizes. Our conclusions are presented in Section 7.

\section{Problem description}

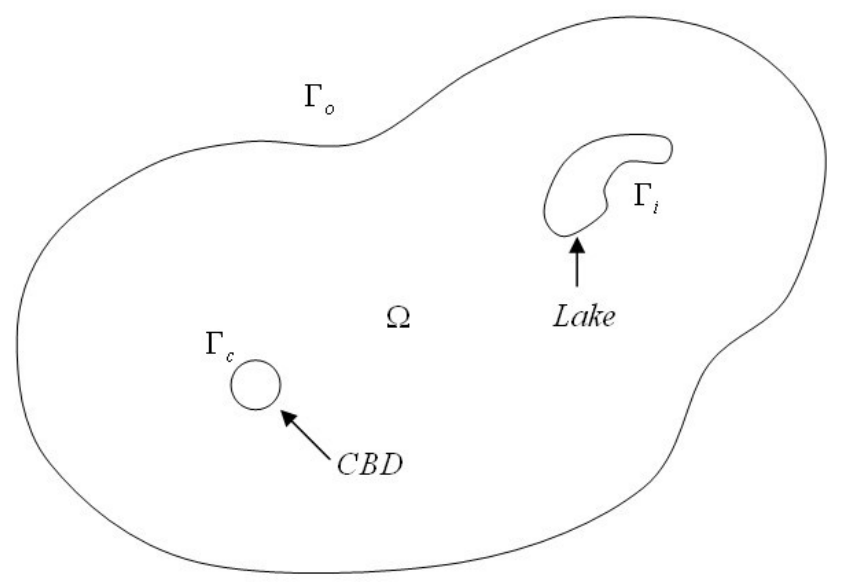

Figure 1: The modeling domain

As Figure 1 shows, the modeled region is an urban city denoted by $\Omega$. Let $\Gamma_{o}$ be the outer boundary of the city, let $\Gamma_{c}$ be the boundary of the compact CBD and let $\Gamma_{i}$ be the boundary of an obstruction such as a lake, park or undeveloped area where traffic is not allowed to enter. Thus, the boundary of $\Omega$ is $\Gamma=\Gamma_{o} \bigcup \Gamma_{c} \cup \Gamma_{i}$. The travelers' homes are continuously located along $(x, y) \in \Omega$. They travel to the CBD within the modeling region for a given time-dependent demand.

The variables are denoted as follows.

- $\rho(x, y, t)$ (in veh/ $\left.\mathrm{km}^{2}\right)$ is the density of travelers at location $(x, y)$ at time $t$. Because we assume that no traveler is allowed to leave the city by crossing the boundary $\Gamma_{o}$ or to enter the obstruction though $\Gamma_{i}$, we have

$$
\rho(x, y, t)=0, \quad \forall(x, y) \in \Gamma_{o} \bigcup \Gamma_{i}, \quad t \in T,
$$


where $T=\left[0, t_{\text {end }}\right]$ (in $h$ ) is the modeling period.

- $\mathbf{v}=(u(x, y, t), v(x, y, t))$ is the velocity vector at location $(x, y)$ at time $t$.

- $U(x, y, t)($ in $\mathrm{km} / \mathrm{h})$ is the speed, which is the norm of the velocity vector, i.e., $U=|\mathbf{v}|$, and is determined by the density as

$$
U(x, y, t)=U_{f} e^{-\beta \rho^{2}}, \quad \forall(x, y) \in \Omega, \quad t \in T
$$

where $U_{f}(x, y)($ in $k m / h)$ is the free-flow speed and $\beta(x, y)\left(\right.$ in $\left.k m^{4} / v e h^{2}\right)$ is a positive scalar related to the road condition.

- $\mathbf{F}=\left(f_{1}(x, y, t), f_{2}(x, y, t)\right)$ is the flow vector at location $(x, y)$ at time $t$, which is defined as

$$
\mathbf{F}=\rho \mathbf{v}, \quad \forall(x, y) \in \Omega, \quad t \in T
$$

- $|\mathbf{F}|$ is the flow intensity, which is the norm of the flow vector $\mathbf{F}$, and is defined as

$$
|\mathbf{F}|=\rho U, \quad \forall(x, y) \in \Omega, \quad t \in T .
$$

As $U$ is a function of $\rho,|\mathbf{F}|$ can also be determined by the density, as shown in Figure 2. Let us define a critical density $\rho_{c}$. When $\rho$ is between 0 and $\rho_{c}$, it is called the non-congested condition. When the density is greater than $\rho_{c}$, it is called the congested condition. $|\mathbf{F}|$ increases as $\rho$ increases from 0 to $\rho_{c}$, and decreases as $\rho$ increases beyond $\rho_{c}$. The maximum flow intensity is achieved at a density of $\rho_{c}$, which is denoted by $F_{\max }$. The flow-density relationship shown in Figure 2 is called the macroscopic fundamental diagram (Daganzo and Geroliminis, 2008; Geroliminis and Daganzo, 2008). Macroscopic fundamental diagrams (MFDs) recently underwent numerous extensions and refinements to enhance their usefulness in representing macroscopic characteristics of dense urban road networks (Daganzo et al., 2011; Gayah and Daganzo, 2011; Geroliminis and Sun, 2011; Geroliminis and Boyaci, 2012). 


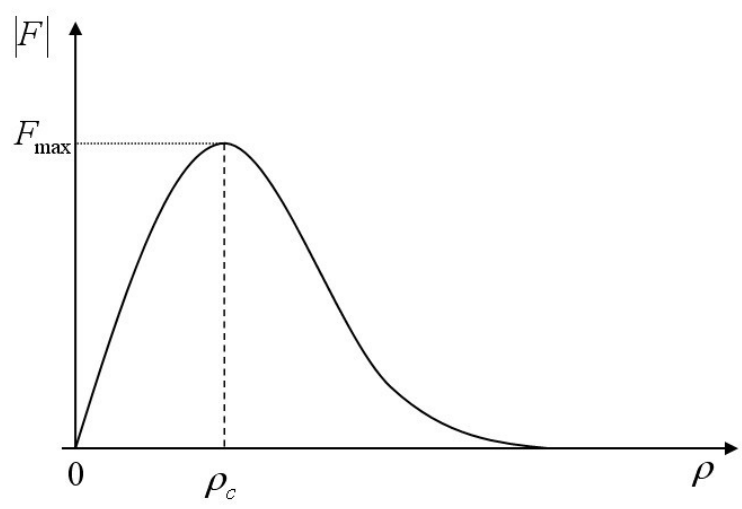

Figure 2: The macroscopic fundamental diagram

- $q(x, y, t)$ (in $\left.v e h / \mathrm{km}^{2} / h\right)$ is the travel demand at location $(x, y)$ at time $t$, which is a non-negative and time-varying function.

- $c(x, y, t)$ (in $\$ / k m)$ is the local travel cost per unit distance of travel at location $(x, y)$ at time $t$, and is defined as

$$
c(x, y, t)=\kappa\left(\frac{1}{U}+\pi(\rho)\right), \quad \forall(x, y) \in \Omega, \quad t \in T,
$$

where $\kappa$ is the value of time, $\frac{\kappa}{U}$ represents the cost associated with the travel time, and $\kappa \pi(\rho)$ represents other costs that are dependent of the density.

- $\phi(x, y, t)$ is the total travel cost incurred by a traveler who departs from location $(x, y)$ at time $t$ to travel to the CBD using the constructed path-choice strategy.

Next, we need to construct a path-choice strategy and a model of this problem to investigate the relationships between the different variables. Here, we assume that the road network is very dense and that travelers can move freely around it. We also assume that travelers have perfect information about traffic conditions over time, and that they will therefore choose the path that minimizes the actual travel cost (not the instantaneous travel cost), resulting in a predictive user equilibrium for a dynamic system. 


\section{Inconsistency of the original PDUO-C model}

\subsection{Original model}

Jiang et al. (2011) constructed a PDUO-C model to solve the problem mentioned previously. In their path-choice strategy, the following two conditions are required:

$$
(u, v, 1) \|-\left(\phi_{x}, \phi_{y}, \phi_{t}\right)
$$

and

$$
|\bar{\nabla} \phi|=\frac{c U}{\sqrt{U^{2}+1}},
$$

where $\|$ means that two vectors are parallel and $\bar{\nabla}=\left(\frac{\partial}{\partial x}, \frac{\partial}{\partial y}, \frac{\partial}{\partial t}\right)$. It has been proved that under these two conditions, travelers choose their path to the CBD in a dynamic predictive user-optimal manner.

The model proposed by Jiang et al. (2011) can be solved by

$$
\left\{\begin{array}{lll}
\rho_{t}+\nabla \cdot \mathbf{F}=q, & \forall(x, y) \in \Omega, & t \in T, \\
\mathbf{F}=-\rho U \frac{\nabla \phi}{\mid \nabla \phi}, & \forall(x, y) \in \Omega, & t \in T, \\
|\nabla \phi|=\frac{c U^{2}}{U^{2}+1}, & \forall(x, y) \in \Omega, & t \in T, \\
\phi_{t}+\frac{1}{U}|\nabla \phi|=0, & \forall(x, y) \in \Omega, \quad t \in T,
\end{array}\right.
$$

subject to the initial boundary conditions

$$
\left\{\begin{array}{lll}
\rho(x, y, 0)=\rho_{0}(x, y), & \forall(x, y) \in \Omega, & \\
\rho(x, y, t)=0, & \forall(x, y) \in \Gamma_{o} \cup \Gamma_{i}, & t \in T, \\
\phi=\phi_{C B D}, & \forall(x, y) \in \Gamma_{c}, & t \in T,
\end{array}\right.
$$

where $\nabla=\left(\frac{\partial}{\partial x}, \frac{\partial}{\partial y}\right)$.

\subsection{The inconsistency}

We first illustrate that Equation (6) (i.e., Equation (10) in the Jiang et al. paper) in the path-choice strategy is inappropriate under certain conditions. Consider a steady state problem, in which the cost $\phi$ is time independent, i.e., $\phi_{t}=0$. In this case, Equation (6) becomes $(u, v, 1) \|\left(\phi_{x}, \phi_{y}, 0\right)$, which is obviously undesirable.

The last equation of the model in Equation (8) is derived from an deficient pathchoice strategy. We can easily see that the units of the two terms in this equation are inconsistent. 
Due to the inconsistency in the path-choice strategy, the whole model (8) is ill-posed mathematically. Note that the first three equations in (8) already represent a well-posed problem if proper initial boundary conditions are given. Hence, the last equation is redundant. We now verify that there exists a solution $(\rho, \phi)$ that satisfies the first three equations, but not the last. Again, consider the steady state problem, in which the density $\rho(x, y, t)=\rho_{0}$, the speed $U(x, y, t)=U_{0}$ and the local cost $c(x, y, t)=c_{0}$ are all positive constants. Take our domain to be a region of arbitrary configurations with a CBD in the center. The boundary of the $\mathrm{CBD}$ (i.e., $\left.\Gamma_{c}\right)$ is a circle located at $(0,0)$ with a diameter of 1 . Given a traffic demand $q(x, y, t)=-\frac{\rho_{0} U_{0}}{\sqrt{x^{2}+y^{2}}}$, we can easily verify that $\phi(x, y, t)=\frac{C_{0} U_{0}^{2}}{U_{0}^{2}+1}\left(\sqrt{x^{2}+y^{2}}-1\right)$ satisfies the first three equations. However, we can show that

$$
\phi_{t}+\frac{1}{U}|\nabla \phi|=\frac{C_{0} U_{0}}{U_{0}^{2}+1}>0
$$

which contradicts the last equation in (8).

\section{An improved model}

As there is an inconsistency in the original PDUO-C model, an improved model is needed to solve the PDUO-C problem. In Section 4.1, we construct a path-choice strategy for the case in which the only cost is the travel time. A general path-choice strategy for a general local cost function is presented in Section 4.2, and Section 4.3 describes the relationship between them. The initial boundary conditions and the complete system of our new model are discussed in Section 4.4.

\subsection{The derivation of the path-choice strategy in the special case that the cost is the time}

First, let us consider the case in which the cost is the travel time in the domain. The path-choice strategy in this paper is discussed in the time-space domain, which is shown in Figure 3. We use the $\mathrm{x}-\mathrm{y}$ plane to represent the space domain in which the vehicles 


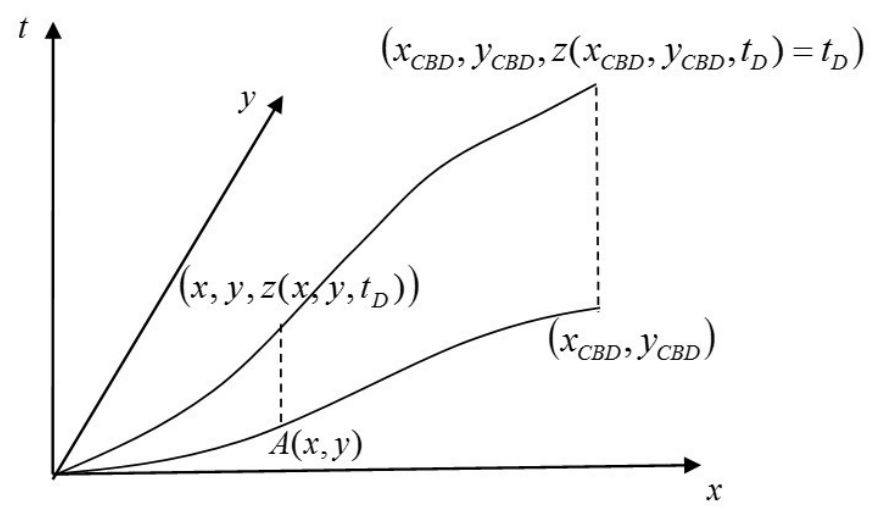

Figure 3: The 3D trajectory of a vehicle

drive. The vertical axis represents the time. Let the coordinate of point $\mathrm{A}$ in the $\mathrm{x}$ $\mathrm{y}$ plane be $(\mathrm{x}, \mathrm{y})$. The curve that passes point $(\mathrm{x}, \mathrm{y}, \mathrm{t})$ represents the $3 \mathrm{D}$ trajectory of a vehicle passing point $\mathrm{A}$ at time $\mathrm{t}$ and heading toward the CBD in the time-space domain.

Definition 1 (The departure time): Suppose the speed vector in the time-space domain is known and denoted by $(u(x, y, t), v(x, y, t))$. We denote $z\left(x, y, t_{D}\right)$ as the departure time at point $(x, y)$, such that a vehicle can arrive at the CBD at time $t_{D}$ along the speed trajectory.

Let us now consider the derivation of the equation to compute $z\left(x, y, t_{D}\right)$. Given a time $t_{D}$, we can view $z\left(x, y, t_{D}\right)$ as a surface in the time-space domain. On this surface, a vehicle can reach the CBD at time $t_{D}$ when moving along the given speed trajectory. We define a path on this surface as $(x(t), y(t), t)$ with the parameter $t$, and the position of the CBD is $\left(x\left(t_{D}\right), y\left(t_{D}\right)\right)$. We clearly have

$$
z\left(x(t), y(t), t_{D}\right)=t
$$

Hence, along this path, we have

$$
\frac{d z}{d t}=\frac{\partial z}{\partial x} \frac{d x}{d t}+\frac{\partial z}{\partial y} \frac{d y}{d t}=1
$$

As $\frac{d x}{d t}=u(x, y, t)=u\left(x, y, z\left(x, y, t_{D}\right)\right)$ and $\frac{d y}{d t}=v(x, y, t)=v\left(x, y, z\left(x, y, t_{D}\right)\right)$, we can 
then obtain the following equation to compute $z\left(x, y, t_{D}\right)$ :

$$
\left\{\begin{array}{l}
\frac{\partial z\left(x, y, t_{D}\right)}{\partial x} u\left(x, y, z\left(x, y, t_{D}\right)\right)+\frac{\partial z\left(x, y, t_{D}\right)}{\partial y} v\left(x, y, z\left(x, y, t_{D}\right)\right)=1, \\
z\left(C B D, t_{D}\right)=t_{D}
\end{array}\right.
$$

For each $t_{D}$, the initial condition is set at time $t_{D}$, and we can solve this equation outwards from the CBD, by backtracking in time.

In the preceding equation, for each given $t_{D}$ and the speed vector $\mathbf{v}=(u(x, y, t), v(x, y, t))$, the corresponding $\nabla z=\left(z_{x}, z_{y}\right)$ needs not be parallel to $\mathbf{v}=(u, v)$. However, in the following theorem, we illustrate our path-choice strategy.

Theorem 1: If we choose the speed vector $\mathbf{v}$ such that the resulting $\nabla z$ is always parallel to $\mathbf{v}$ and has the same direction, then the dynamic predictive user equilibrium in terms of total travel time to the CBD is satisfied.

Proof: See Appendix A.

With the condition that $\nabla z \| \mathbf{v}$, we can simplify Equation (13) as

$$
\left\{\begin{array}{l}
|\nabla z|=1 / U \\
z\left(C B D, t_{D}\right)=t_{D}
\end{array}\right.
$$

\subsection{The derivation of the path-choice formula with a more gen- eral cost}

So far, we have used the total travel time to represent the cost. We must now construct a more general path-choice strategy for a general local cost function in the time-space domain.

If the speed vector $(u(x, y, t), v(x, y, t))$ in the time-space domain is known, then the cost potential function $\phi(x, y, t)$ can be determined (discussed in more detail later) and $\left(\phi_{x}, \phi_{y}\right)$ can be obtained. $(u, v)$ can be arbitrary, and need not be parallel with $\left(\phi_{x}, \phi_{y}\right)$. However, in the following theorem, we show that if we choose the vector $(u, v)$ such that the resulting $-\left(\phi_{x}, \phi_{y}\right)$ is parallel to $(u, v)$, then the dynamic user equilibrium is satisfied.

Theorem 2: If $(u, v) \|\left(-\phi_{x},-\phi_{y}\right)$, then the predictive dynamic user equilibrium principle is satisfied. 
Proof: See Appendix B.

We compute $\phi(x, y, t)$ when the speed vector in the time-space domain is given. In fact, we only need to choose the vector $(u, v)$ such that the resulting $-\left(\phi_{x}, \phi_{y}\right)$ is parallel to $(u, v)$. We define a path along this given speed vector as $(x(t), y(t), t)$ with parameter t. Along this path, we have

$$
\begin{aligned}
\frac{d \phi}{d t} & =\phi_{x} \frac{d x(t)}{d t}+\phi_{y} \frac{d y(t)}{d t}+\phi_{t} \\
& =\phi_{x} u+\phi_{y} v+\phi_{t} \\
& =-|\nabla \phi| U+\phi_{t} .
\end{aligned}
$$

By the definition of the derivative, we also have

$$
\begin{aligned}
\frac{d \phi}{d t} & =\lim _{\Delta t \rightarrow 0} \frac{\phi(x(t+\Delta t), y(t+\Delta t), t+\Delta t)-\phi(x(t), y(t), t)}{\Delta t} \\
& =-U(x(t), y(t), t) \lim _{\Delta t \rightarrow 0} \frac{\phi(x(t), y(t), t)-\phi(x(t+\Delta t), y(t+\Delta t), t+\Delta t)}{\Delta t U(x(t), y(t), t)} \\
& =-U(x, y, t) c(x, y, t) .
\end{aligned}
$$

By combining Equations (15) and (16), we obtain the equation to solve $\phi$ :

$$
\frac{1}{U} \phi_{t}-|\nabla \phi|=-c
$$

which is a Hamilton-Jacobi equation.

\subsection{The relationship between the special case and the general case}

In Section 4.1, we considered the case in which the cost is the travel time in the domain. An important question is whether this is a special case of the general strategy in Section 4.2. If the answer is yes, we will have more confidence in our new model.

In Section 4.1 , we denoted $z\left(x, y, t_{D}\right)$ as the departure time from point $(x, y)$ such that the vehicle can arrive at the CBD at time $t_{D}$. Thus, in the special case in which the cost $\phi(x, y, t)$ is the travel time from point $(x, y, t)$ to the CBD, we have

$$
\phi\left(x, y, z\left(x, y, t_{D}\right)\right)=t_{D}-z\left(x, y, t_{D}\right)
$$


We can then obtain

$$
\left\{\begin{array}{l}
\phi_{x}+\phi_{t} \cdot z_{x}=-z_{x} \\
\phi_{y}+\phi_{t} \cdot z_{y}=-z_{y}
\end{array}\right.
$$

i.e.,

$$
\left\{\begin{array}{l}
\phi_{x}=-\left(\phi_{t}+1\right) z_{x} \\
\phi_{y}=-\left(\phi_{t}+1\right) z_{y}
\end{array}\right.
$$

Before we continue, let us consider the following theorem.

Theorem 3: In the case that the cost $\phi(x, y, t)$ is the travel time in the domain, we have $\phi_{t} \geq-1$

Proof: See Appendix C.

Using Equation (20) and Theorem 3, we can obtain $-\left(\phi_{x}, \phi_{y}\right) \|\left(z_{x}, z_{y}\right)$. Hence, the requirement of $(u, v) \|-\left(\phi_{x}, \phi_{y}\right)$ in Section 4.2 and the requirement of $(u, v) \|\left(z_{x}, z_{y}\right)$ in Section 4.1 are the same.

In Section 4.2, the formula to compute $\phi$ is

$$
\frac{1}{U} \phi_{t}-|\nabla \phi|=-c
$$

In the case that the travel cost is the travel time in the domain, the local cost is in fact the travel time per unit distance of movement, i.e.,

$$
c(x, y, t)=1 / U(x, y, t) .
$$

Using Equations (20) and (22) and Theorem 3, we can simplify Equation (21) and obtain

$$
|\nabla z|=\frac{1}{U}
$$

which is the same as the formula in Section 4.1.

\subsection{The complete model and the discussion of initial boundary conditions}

So far, we have constructed a general path-choice strategy. In this section, we present some proper equations and their initial boundary conditions to construct a complete model using this new path-choice strategy. 


\subsubsection{The conservation law and its initial boundary conditions}

Similar to flow conservation in fluid dynamics, the density $\rho(x, y, t)$ is governed by the following flow conservation law:

$$
\rho_{t}+\nabla \cdot \mathbf{F}=q, \quad \forall(x, y) \in \Omega, t \in T
$$

where $\rho_{t}=\frac{\partial \rho}{\partial t}$ and $\nabla=\left(\frac{\partial}{\partial x}, \frac{\partial}{\partial y}\right)$. Using the path-choice strategy in Section 4.2, we know that if we choose the vector $\mathbf{v}=(u, v)$ such that the resulting $-\left(\phi_{x}, \phi_{y}\right)$ is parallel to $(u, v)$, then the dynamic user equilibrium is satisfied. Hence, we have

$$
\mathbf{F}=\rho \mathbf{v}=-\rho U \frac{\nabla \phi}{|\nabla \phi|}
$$

Assuming that no vehicle is allowed to enter the obstruction through the boundary $\Gamma_{i}$ or leave the city through $\Gamma_{o}$, we have the boundary condition

$$
\rho(x, y, t)=0, \quad \forall(x, y) \in \Gamma_{o} \bigcup \Gamma_{i}, t \in T
$$

We set the initial time as $t=0$ and let $\rho_{0}(x, y)$ be the density of traffic at location $(x, y)$ at the beginning of the modeling period. As such, the initial condition is

$$
\rho(x, y, 0)=\rho_{0}(x, y), \quad \forall(x, y) \in \Omega
$$

\subsubsection{The Hamilton-Jacobi equation and its initial boundary conditions}

To solve the aforementioned conservation law, we must determine the travel cost $\phi$ to compute the flow vector in Equation (25). The travel cost can be solved by the HamiltonJacobi equation, which is presented in Section 4.2:

$$
\frac{1}{U} \phi_{t}-|\nabla \phi|=-c, \quad \forall(x, y) \in \Omega, t \in T
$$

Let

$$
\phi(x, y, t)=\phi_{C B D}, \quad \forall(x, y) \in \Gamma_{c}, t \in T
$$

which represents the boundary value of $\phi$ on $\Gamma_{c}$ and can be interpreted as the cost to the traveler of entering the CBD. 
There is still the problem of how to set the initial condition. Note that the travel cost to the CBD only depends on the events that will occur in the future, and has nothing to do with events that happened in the past. Hence, it seems reasonable to solve the Hamilton-Jacobi equation along the negative time direction, and thus we set the initial time at $t=t_{\text {end }}$. Here, we assume that all travelers have entered the CBD and that there is no traffic in the city at $t=t_{\text {end }}$. This can be considered as a static state, and the travel cost to the CBD is the instantaneous cost. Following Huang et al. (2009), we use a $2 \mathrm{D}$ Eikonal equation to solve the initial values $\phi_{0}(x, y)$ :

$$
\begin{cases}\left|\nabla \phi_{0}(x, y)\right|=c\left(x, y, t_{\text {end }}\right) & \forall(x, y) \in \Omega, \\ \phi_{0}(x, y)=\phi_{C B D}, & \forall(x, y) \in \Gamma_{c} .\end{cases}
$$

\subsubsection{The complete model}

We can write our model in two parts.

\section{The $C L$ part is}

$$
\begin{cases}\rho_{t}+\nabla \cdot \mathbf{F}=q, & \forall(x, y) \in \Omega, \quad t \in T, \\ \mathbf{F}=-\rho U \frac{\nabla \phi}{|\nabla \phi|}, & \forall(x, y) \in \Omega, \quad t \in T, \\ \rho(x, y, t)=0, & \forall(x, y) \in \Gamma_{o} \cup \Gamma_{i}, \quad t \in T, \\ \rho(x, y, 0)=\rho_{0}(x, y), & \forall(x, y) \in \Omega .\end{cases}
$$

The $H J$ part is

$$
\begin{cases}\frac{1}{U} \phi_{t}-|\nabla \phi|=-c, & \forall(x, y) \in \Omega, \quad t \in T, \\ \phi(x, y, t)=\phi_{C B D}, & \forall(x, y) \in \Gamma_{c}, \quad t \in T, \\ \phi\left(x, y, t_{\text {end }}\right)=\phi_{0}(x, y), & \forall(x, y) \in \Omega,\end{cases}
$$

where the initial value $\phi_{0}(x, y)$ is computed by a $2 \mathrm{D}$ Eikonal equation:

$$
\begin{cases}\left|\nabla \phi_{0}(x, y)\right|=c\left(x, y, t_{\text {end }}\right), & \forall(x, y) \in \Omega, \\ \phi_{0}(x, y)=\phi_{C B D}, & \forall(x, y) \in \Gamma_{c} .\end{cases}
$$

Here, $\rho(x, y, t)$ is governed by the conservation law, and $\phi(x, y, t)$ is computed using the Hamilton-Jacobi equation. Note that whereas the initial time in the conservation law is set as $t=0$, the initial time in the Hamilton-Jacobi equation is $t=t_{\text {end }}$. 


\section{Solution algorithm}

In this section, we describe the solution algorithms used in the improved model, such as the Lax-Friedrichs scheme used to solve the conservation law Equation (31) and Hamilton-Jacobi Equation (32), the fast sweeping method used to solve the Eikonal Equation (33) and the self-adaptive MSA used to solve the fixed-point problem.

\subsection{Lax-Friedrichs scheme used to solve the conservation law}

In this subsection, we assume that the cost potential function $\phi(x, y, t)$ is known for all $(x, y) \in \Omega$ and $t \in T$, and focus on the numerical method to solve the conservation law:

$$
\begin{cases}\rho_{t}+\nabla \cdot \mathbf{F}=q, & \forall(x, y) \in \Omega, \quad t \in T, \\ \mathbf{F}=-\rho U \frac{\nabla \phi}{|\nabla \phi|}, & \forall(x, y) \in \Omega, \quad t \in T, \\ \rho(x, y, t)=0, & \forall(x, y) \in \Gamma_{o} \cup \Gamma_{i}, \quad t \in T, \\ \rho(x, y, 0)=\rho_{0}(x, y), & \forall(x, y) \in \Omega .\end{cases}
$$

We use the conservative difference scheme to approximate the point values $\rho_{i, j}^{n} \approx$ $\rho\left(x_{i}, y_{j}, t^{n}\right):$

$$
\rho_{i, j}^{n+1}=\rho_{i, j}^{n}-\frac{\Delta t}{\Delta x}\left(\hat{f}_{i+\frac{1}{2}, j}-\hat{f}_{i-\frac{1}{2}, j}\right)-\frac{\Delta t}{\Delta y}\left(\hat{g}_{i, j+\frac{1}{2}}-\hat{g}_{i, j-\frac{1}{2}}\right)+q_{i, j} \Delta t
$$

where $q_{i, j}=q\left(x_{i}, y_{j}, t^{n}\right)$ is the given demand at location $\left(x_{i}, y_{j}\right)$ at time $t^{n} ; \Delta x$ and $\Delta y$ are the mesh sizes in the $x$ and $y$ directions, respectively and $\hat{f}_{i+\frac{1}{2}, j}$ and $\hat{g}_{i, j+\frac{1}{2}}$ are numerical fluxes in the $x$ and $y$ directions, respectively. Here, we use the Lax-Friedrichs flux, which is a monotone flux:

$$
\begin{aligned}
& \hat{f}_{i+\frac{1}{2}, j}=\frac{1}{2}\left[f\left(\rho_{i, j}^{n}\right)+f\left(\rho_{i+1, j}^{n}\right)-\alpha_{f}\left(\rho_{i+1, j}^{n}-\rho_{i, j}^{n}\right)\right] \\
& \hat{g}_{i, j+\frac{1}{2}}=\frac{1}{2}\left[g\left(\rho_{i, j}^{n}\right)+g\left(\rho_{i, j+1}^{n}\right)-\alpha_{g}\left(\rho_{i, j+1}^{n}-\rho_{i, j}^{n}\right)\right],
\end{aligned}
$$

where $\alpha_{f}=\max \left|f^{\prime}(\rho)\right|$ and $\alpha_{g}=\max \left|g^{\prime}(\rho)\right|$. 


\subsection{Lax-Friedrichs scheme used to solve the time-dependent Hamilton-Jacobi equation}

In this subsection, we suppose that the density $\rho(x, y, t)$ is known for all $(x, y) \in \Omega$ and $t \in T$, and focus on the numerical method to solve the Hamilton-Jacobi equation:

$$
\begin{cases}\frac{1}{U} \phi_{t}-|\nabla \phi|=-c, & \forall(x, y) \in \Omega, \quad t \in T, \\ \phi(x, y, t)=\phi_{C B D}, & \forall(x, y) \in \Gamma_{c}, \quad t \in T, \\ \phi\left(x, y, t_{\text {end }}\right)=\phi_{0}(x, y), & \forall(x, y) \in \Omega .\end{cases}
$$

Note that the initial time is $t=t_{\text {end }}$ and that the initial value $\phi_{0}(x, y)$ is computed by the Eikonal Equation (33). We give the numerical method for solving the Eikonal equation in Appendix D, and in this subsection we assume that $\phi_{0}(x, y)$ is known.

As the initial time is $t=t_{\text {end }}$, we define

$$
\tau=t_{\text {end }}-t, \Phi(x, y, \tau)=\phi\left(x, y, t_{\text {end }}-\tau\right)
$$

and thus we rewrite the time-dependent HJ equation into the usual form:

$$
\begin{cases}\frac{1}{U} \Phi_{\tau}+|\nabla \Phi|=c, & \forall(x, y) \in \Omega, \quad \tau \in T, \\ \Phi(x, y, \tau)=\phi_{C B D}, & \forall(x, y) \in \Gamma_{c}, \quad \tau \in T, \\ \Phi(x, y, 0)=\phi_{0}(x, y), & \forall(x, y) \in \Omega .\end{cases}
$$

When we define

$$
H\left(\Phi_{x}, \Phi_{y}\right)=U(|\nabla \Phi|-c)
$$

then the scheme to solve $\Phi_{\tau}+H\left(\Phi_{x}, \Phi_{y}\right)=0$ is

$$
\Phi_{i, j}^{n+1}=\Phi_{i, j}^{n}-\Delta t \hat{H}\left(\left(\Phi_{x}\right)_{i, j}^{-},\left(\Phi_{x}\right)_{i, j}^{+},\left(\Phi_{y}\right)_{i, j}^{-},\left(\Phi_{y}\right)_{i, j}^{+}\right)
$$

with

$$
\begin{aligned}
\left(\Phi_{x}\right)_{i, j}^{-}=\frac{\Phi_{i, j}-\Phi_{i-1, j}}{\Delta x}, & \left(\Phi_{x}\right)_{i, j}^{+}=\frac{\Phi_{i+1, j}-\Phi_{i, j}}{\Delta x}, \\
\left(\Phi_{y}\right)_{i, j}^{-}=\frac{\Phi_{i, j}-\Phi_{i, j-1}}{\Delta y}, & \left(\Phi_{y}\right)_{i, j}^{+}=\frac{\Phi_{i, j+1}-\Phi_{i, j}}{\Delta y},
\end{aligned}
$$

where $\hat{H}$ is a Lipschitz continuous monotone flux consistent with $H$. Here, we use the global Lax-Friedrichs flux:

$$
\hat{H}\left(u^{-}, u^{+}, v^{-}, v^{+}\right)=H\left(\frac{u^{-}+u^{+}}{2}, \frac{v^{-}+v^{+}}{2}\right)-\frac{1}{2} \alpha^{x}\left(u^{+}-u^{-}\right)-\frac{1}{2} \alpha^{y}\left(v^{+}-v^{-}\right),
$$


where $\alpha^{x}$ and $\alpha^{y}$ are the viscosity constants and are defined as

$$
\alpha^{x}=\max _{A \leq u \leq B, C \leq v \leq D}\left|H_{1}(u, v)\right|, \quad \alpha^{y}=\max _{A \leq u \leq B, C \leq v \leq D}\left|H_{2}(u, v)\right| .
$$

$H_{1}\left(H_{2}\right)$ is the partial derivative of $H$ in terms of $\Phi_{x}\left(\Phi_{y}\right),[A, B]$ is the value range of $u^{ \pm}$and $[C, D]$ is the value range of $v^{ \pm}$.

\subsection{Fixed-point problem and MSA}

Note that the two parts of the model are closely interconnected. When computing the density $\rho$ by solving the CL (31), we must know the total cost $\phi$ of getting to the CBD from every point, and thus we can decide on the flow direction needed to compute the density at the next time level. When computing the cost $\phi$ by solving the HJ (32), we need the density information to obtain the local cost. However, neither $\rho$ nor $\phi$ is known in advance, and these two equations cannot be solved together as they have different initial times. As mentioned in the introduction, this model is in fact a fixed-point problem that can be solved by the MSA. We illustrate this in detail in this subsection.

Define the vector of the numerical solutions at each grid point and each time level as

$$
\begin{gathered}
\vec{\rho}=\left\{\rho_{i, j}^{n}, i=1, \cdots, N_{x}, j=1, \cdots, N_{y}, n=1, \cdots, N_{t}\right\} \\
\vec{\phi}=\left\{\phi_{i, j}^{n}, i=1, \cdots, N_{x}, j=1, \cdots, N_{y}, n=1, \cdots, N_{t}\right\}
\end{gathered}
$$

where $N_{x}, N_{y}$ and $N_{t}$ are the numbers of grid points in x, y and t, respectively. Let us now give the definition for one iteration, which contains two steps, as shown in Figure 4.

Step 1. With a given vector $\vec{\phi}^{\text {old }}$, we solve the CL (31) from $t=0$ to $t=t_{\text {end }}$ using the Lax-Friedrichs scheme described in Section 5.1, and thus obtain the vector $\vec{\rho}$. We denote this step as

$$
\vec{\rho}=\mathbf{g}\left(\vec{\phi}^{o l d}\right)
$$

Step 2. Solve the HJ Equation (32) from $t=t_{\text {end }}$ to $t=0$ using the Lax-Friedrichs scheme described in Section 5.2 to obtain an updated vector $\vec{\phi}^{n e w}$. We denote this step 


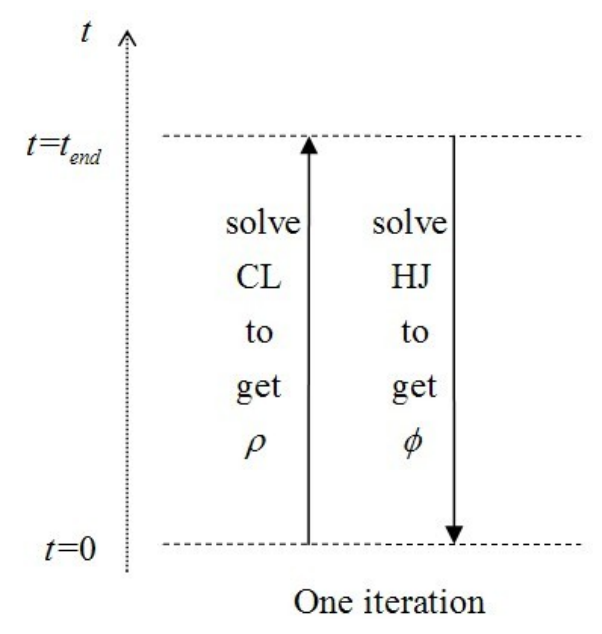

Figure 4: Definition of one iteration

as

$$
\vec{\phi}^{n e w}=\mathbf{h}(\vec{\rho})
$$

We consider Steps 1 and 2 as one iteration and denote it as

$$
\vec{\phi}^{\text {new }}=\mathbf{h}\left(\mathbf{g}\left(\vec{\phi}^{\text {old }}\right)\right)=\mathbf{f}\left(\vec{\phi}^{\text {old }}\right)
$$

With the definition of one iteration and the function $\mathbf{f}$, the model translates to a fixedpoint problem

$$
\vec{\phi}=\mathbf{f}(\vec{\phi})
$$

which can be solved by the MSA.

The MSA was first introduced by Robbins and Monro (1951) to solve the fixed-point problem. A general MSA is an iterative process. If we denote the solution before the $k$ th iteration as $\vec{\phi}^{k}$, then computing the MSA to obtain $\vec{\phi}^{k+1}$ involves the following steps: Step 1. Solve a temporary solution $\vec{y}^{k}=\mathbf{f}\left(\vec{\phi}^{k}\right)$ during the $k$ th iteration.

Step 2. Choose a step size $\lambda_{k}$ and use the following equation to obtain $\vec{\phi}^{k+1}$ :

$$
\vec{\phi}^{k+1}=\left(1-\lambda_{k}\right) \vec{\phi}^{k}+\lambda_{k} \cdot \vec{y}^{k}, k=1,2, \cdots
$$

Convergence is declared if

$$
\left\|\overrightarrow{\phi^{k+1}}-\vec{\phi}^{k}\right\| \leq \delta
$$


where $\delta$ is a given convergence threshold value. We use $\delta=10^{-2}$ and $L_{2}$ as the norm in our computation.

The selection of the step size sequence $\left\{\lambda_{k}\right\}$ is important to guaranteeing the convergence and efficiency of this method. In the conventional MSA, Robbins and Monro (1951) suggested a predetermined step size sequence of $\left\{\lambda_{k}=1 / k\right\}$. Performing the MSA with this step size sequence has two main weaknesses: i) the step sizes for the first few iterations are very large, which may amplify the error and require more iterations to reduce it, and ii) the step sizes become too small after a large number of iterations, such that the weight of $y^{k}$ is too small in the computation of $x^{k+1}$ and the convergence speed is extremely slow. Hence, the conventional MSA often requires a large number of iterations to reach a convergent solution. To overcome these two weaknesses, some other methods consider the information generated from the iteration procedure and adjust the step size accordingly. For example, Liu et al. (2009) constructed a self-regulated averaging method by slowing down the decrease in $\left\{\lambda_{k}\right\}$ when current iterations converged and speeding up the decrease in $\left\{\lambda_{k}\right\}$ when the solutions diverged. While this method is much faster than the conventional MSA, the convergence speed still becomes extremely slow as long as the step sizes continue decreasing.

Bar-Gera and Boyce (2006) constructs a type of MSA with a constant step size sequence, efficiently solving travel forecasting problems. It assumes that $\mathbf{f}$ is smooth, and derives the following formula:

$$
\frac{\left\|\vec{\phi}^{k+1}-\mathbf{f}\left(\vec{\phi}^{k+1}\right)\right\|^{2}}{\left\|\vec{\phi}^{k}-\mathbf{f}\left(\vec{\phi}^{k}\right)\right\|^{2}} \rightarrow r^{*}(\lambda),
$$

where $\lambda$ is the constant step size and $r^{*}(\lambda)$ is a convex quadratic function that is pointing up. In addition, $r^{*}(0)=1$ and $\frac{\partial r^{*}}{\partial \lambda}(0)<0$. Any constant step size such that $r^{*}(\lambda)<1$ results in convergence. There specially exists an optimal constant step size $0<\lambda^{*} \leq 1$ such that $r^{*}\left(\lambda^{*}\right)=\min _{\lambda} r^{*}(\lambda)$. However, because the constant step size is related to the properties of $\mathbf{f}(\vec{\phi})$, some prior information is required to determine the step size. Bar-Gera and Boyce (2006) did not offer a general method for determining the optimal 
constant step size for different problems.

In the next subsection, we construct a self-adaptive MSA based on Equation (55) derived by Bar-Gera and Boyce (2006), and automatically determine the optimal step size for the MSA using the least squares method without prior information.

\subsection{A self-adaptive MSA}

Based on Formula (55), we can use the least squares method to fit the curve $r^{*}(\lambda)$, and set the minimum point $\lambda^{*}$ as the updated step size. The procedure for determining the optimal step size used in the self-adaptive MSA is as follows.

1. For the first several iterations, we use the predetermined step sizes

$$
\lambda_{1}=1.0, \lambda_{2}=0.4, \lambda_{3}=0.3, \lambda_{4}=0.2, \lambda_{5}=0.15, \lambda_{6}=0.1, \lambda_{7}=0.05 .
$$

2. After the $k$ th iteration $(k>2)$, we record the step size $\lambda_{k-1}$ used before this iteration and the resulting ratio of the error

$$
r_{k-1}=\frac{\left\|\overrightarrow{\phi^{k}}-\mathbf{f}\left(\vec{\phi}^{k}\right)\right\|^{2}}{\left\|\vec{\phi}^{k-1}-\mathbf{f}\left(\vec{\phi}^{k-1}\right)\right\|^{2}},
$$

thus constituting a discrete point $\left(\lambda_{k-1}, r_{k-1}\right)$ to fit the curve $r^{*}(\lambda)$.

3. For the $n+1(n \geq 7)$ step size, we use the least squares method to fit the discrete points $\left(\lambda_{k}, r_{k}\right), k=1, \cdots, n$ and obtain the fitted quadratic curve $r^{*}(\lambda)$. Define the new step size $\lambda_{n+1}$ as the minimum point of $r^{*}(\lambda)$, i.e., $r^{*}\left(\lambda_{n+1}\right)=\min _{\lambda} r^{*}(\lambda)$.

4. If the new step size is invalid, such that $\lambda_{n+1} \leq 0$ or $\lambda_{n+1} \geq 1$, then we abandon it and set $\lambda_{n+1}=0.5 \lambda_{n}$.

There are several points to note about the self-adaptive MSA.

- The quadratic curve $r^{*}(\lambda)$ must pass though the point $(0,1)$, i.e.,

$$
r^{*}(0)=1,
$$

and as such only two parameters must be determined to fit the curve. 
- Because the accuracy of the discrete points $\left(\lambda_{k}, r_{k}\right)$ is low for the first several iterations due to the nonlinearity of $\mathbf{f}$, we do not use the least squares method until the eighth step.

- We distribute the first several step sizes $\lambda_{k}, k=2, \cdots, 7$ on the interval $[0,0.4]$ to avoid concentrating the step size distribution into too narrow a range, which may lead to a poor least squares estimate.

- The descent speed of $\lambda_{k}, k=1, \cdots, 7$ is much faster than the conventional step size $\lambda_{k}=1 / k$ (Robbins and Monro, 1951) and hence avoids too large an amplification of the error.

- $\lambda_{n+1}=0.5 \lambda_{n}$ is reset when $\lambda_{n+1} \leq 0$ or $\lambda_{n+1} \geq 1$ to avoid trying the same nonoptimal step size repeatedly, which would not produce a better estimate of the quadratic curve. A small step size can also guarantee convergence.

- The self-adaptive MSA uses the information from the iterations and adjusts the step size accordingly. The step size may be inaccurate at the beginning, but becomes increasingly accurate as the number of iterations, $k$, increases. The numerical result shows that the step size is almost a constant after a number of iterations.

\subsection{Solution procedure}

The question of how to set the initial value for the self-adaptive MSA remains. To start, let us compute the density along the positive time direction. While we have no information about the actual total cost of traveling to the CBD, we can compute the instantaneous total cost to the destination at each time level by solving a 2D Eikonal equation, and use the route strategy that satisfies the reactive dynamic user equilibrium principle (Huang et al., 2009). Hence, we can compute the density $\rho(x, y, t)$ by solving 


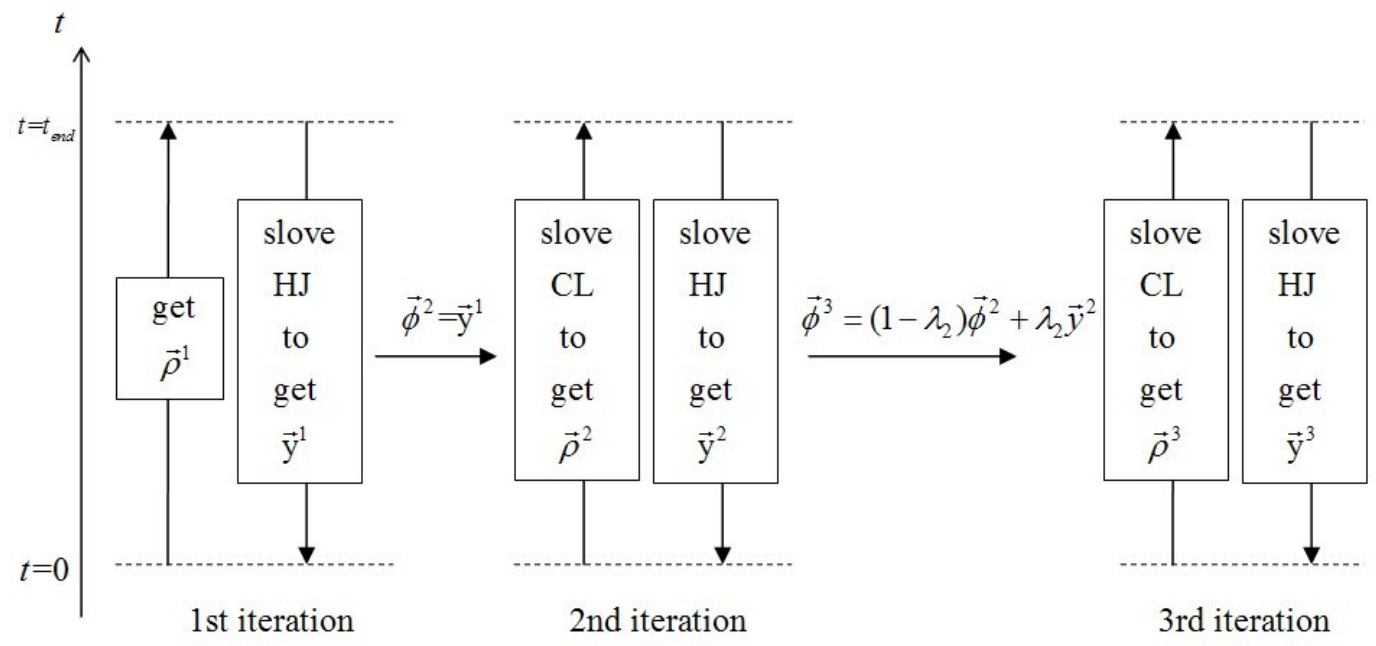

Figure 5: Solution procedure

the following model:

$$
\left\{\begin{array}{lll}
\rho_{t}+\nabla \cdot \mathbf{F}=q, & \forall(x, y) \in \Omega, & t \in T, \\
\mathbf{F}=-\rho U \frac{\nabla \phi}{|\nabla \phi|}, & \forall(x, y) \in \Omega, \quad t \in T, \\
|\nabla \phi|=c(x, y, t), & \forall(x, y) \in \Omega, \quad t \in T,
\end{array}\right.
$$

subject to the initial boundary conditions

$$
\begin{cases}\rho(x, y, t)=0, & \forall(x, y) \in \Gamma_{o} \cup \Gamma_{i}, \quad t \in T, \\ \rho(x, y, 0)=\rho_{0}(x, y), & \forall(x, y) \in \Omega, \\ \phi(x, y, t)=\phi_{C B D}, & \forall(x, y) \in \Gamma_{c}, \quad t \in T\end{cases}
$$

We then obtain the density vector based on the reactive dynamic user equilibrium principle and denote it as $\vec{\rho}^{1}$. We can compute the actual total cost to the CBD by solving HJ Equation (32), which requires backtracking in time to obtain $\vec{y}^{1}=\mathbf{h}\left(\vec{\rho}^{1}\right)$, and set $\vec{\phi}^{2}=\vec{y}^{1}$.

The solution procedure is as follows.

1. Compute the density $\vec{\rho}^{1}$ from $t=0$ to $t=t_{\text {end }}$ using Equation (59). Computing the actual total cost

$$
\vec{y}^{1}=\mathbf{h}\left(\vec{\rho}^{1}\right)
$$

from $t=t_{\text {end }}$ to $t=0$ by solving the HJ Equation (32), we obtain $\vec{\phi}^{2}=\left(1-\lambda_{1}\right) \vec{\phi}^{1}+$ $\lambda_{1} \vec{y}^{1}=\vec{y}^{1}$. 
2. Use the $k$ th solution vector $\vec{\phi}^{k}$ to complete the $k$ th iteration, $k=2, \cdots$, i.e.,

$$
\left\{\begin{array}{l}
\vec{\rho}^{k}=\mathbf{g}\left(\vec{\phi}^{k}\right), \\
\vec{y}^{k}=\mathbf{h}\left(\vec{\rho}^{k}\right)=\mathbf{f}\left(\vec{\phi}^{k}\right) .
\end{array}\right.
$$

3. Compute the step size $\lambda_{k}(k>7)$ using the method described in Section 5.4. The step sizes $\lambda_{k}, k=1, \cdots, 7$ are predetermined.

4. Compute the $(k+1)$ th solution vector

$$
\vec{\phi}^{k+1}=\left(1-\lambda_{k}\right) \vec{\phi}^{k}+\lambda_{k} \cdot \vec{y}^{k}
$$

5. Stop the iteration process when (54) is satisfied.

\section{$6 \quad$ Numerical examples}

To demonstrate the effectiveness of the improved model and the proposed algorithm, we present a numerical example in this section and compare the result with the conventional MSA (Robbins and Monro, 1951) and the self-regulated averaging method (Liu et al., 2009).

\subsection{Problem setting}

As shown in Figure 6, we consider a rectangular domain that is $35 \mathrm{~km}$ long and $25 \mathrm{~km}$ wide in the numerical computation. The center of the compact CBD is located at (10 $\mathrm{km}, 10 \mathrm{~km})$, and a lake is located at $(25 \mathrm{~km}, 15 \mathrm{~km})$. We assume that there is no traffic at the beginning or the end of the modeling period (i.e., $\rho_{0}(x, y)=0, \forall(x, y) \in \Omega$ ), and that no cost is incurred by entering the $\operatorname{CBD}\left(\phi_{C B D}=0, \forall(x, y) \in \Gamma_{c}, t \in T\right)$. We set $t_{\text {end }}=6$, and so the modeling period is $T=[0 h, 6 h]$. The traffic demand function $q$ is defined as

$$
q(x, y, t)=q_{\max }\left[1-\gamma_{1} d(x, y)\right] g(t)
$$

where $q_{\max }=240 v \mathrm{eh} / \mathrm{km}^{2} / \mathrm{h}$ is the maximum demand, $\gamma_{1}=0.01 \mathrm{~km}^{-1}$ is a positive scalar and $d(x, y)=\sqrt{(x-10)^{2}+(y-10)^{2}}$ is the distance from location $(x, y)$ to the 


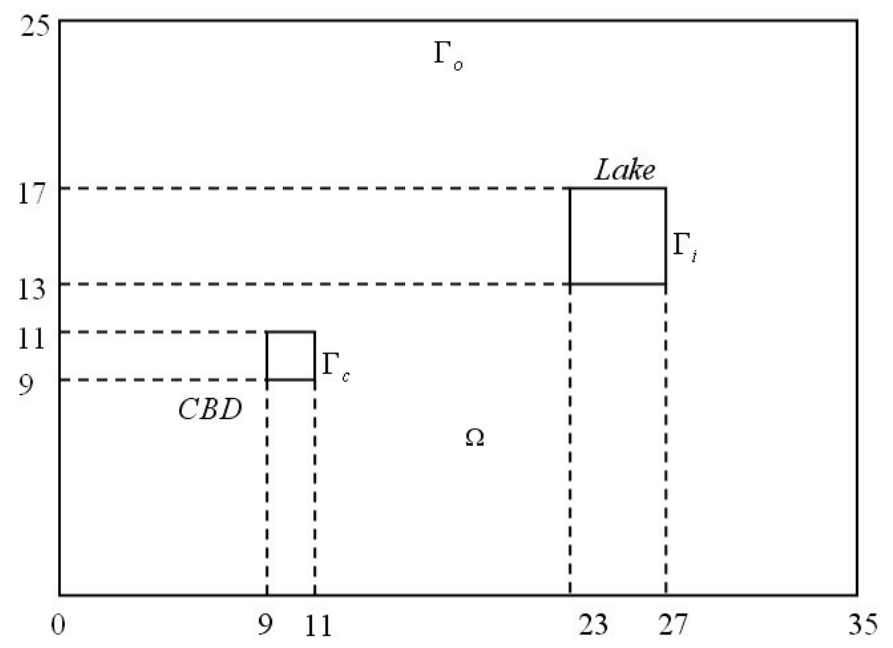

Figure 6: The modeling domain

center of the CBD. The factor $\left[1-\gamma_{1} d(x, y)\right]$ is used to express the higher traffic demand generated in the domain closer to the $\mathrm{CBD}$, where more of the population is located. $g(t)$ is a non-negative and time-varying function defined by

$$
g(t)= \begin{cases}t, & t \in[0 h, 1 h], \\ 1, & t \in[1 h, 2 h], \\ -\frac{4}{5}(t-3)+\frac{1}{5}, & t \in[2 h, 3 h], \\ \frac{1}{5}, & t \in[3 h, 5 h], \\ 0, & t \in[5 h, 6 h] .\end{cases}
$$

The speed function is defined as $U(x, y, t)=U_{f} e^{-\beta \rho^{2}}$, in which $\beta=2 \times 10^{-6} \mathrm{~km}^{4} / v e h^{2}$ and $U_{f}(x, y)$ is the free-flow speed given by

$$
U_{f}(x, y)=U_{\max }\left[1+\gamma_{2} d(x, y)\right]
$$

where $U_{\max }=56 \mathrm{~km} / \mathrm{h}$ is the maximum speed and $\gamma_{2}=4 \times 10^{-3} \mathrm{~km}^{-1}$. The factor $\left[1+\gamma_{2} d(x, y)\right]$ is used to express the faster free-flow speed in the domain far from the CBD, where there are fewer junctions. With this definition, we can compute the critical density of $\rho_{c}$ as $500 \mathrm{veh} / \mathrm{km}^{2}$. The local travel cost per unit of distance is defined as $c(x, y, t)=\kappa\left(\frac{1}{U}+\pi(\rho)\right)$, where $\kappa=90 \$ / h$ and $\pi(\rho)=10^{-8} \rho^{2}$. Because we assume that the capacity of the CBD is large enough to accommodate all of the travelers in the city, $|\mathbf{F}|$ should maintain the maximum flow intensity $F_{\max }$ at the CBD boundary under the 
congested condition.

We now use the composed algorithm described in the previous section to perform the numerical simulation. A uniform mesh with an $N_{x} \times N_{y}$ grid is used. The mesh grids inside the CBD and the mesh grids inside the lake are not computed. The numerical boundary conditions are summarized as follows.

1. On the solid wall boundaries, i.e., the outer boundary of the city, $\Gamma_{o}$, and the boundary of the obstruction, $\Gamma_{i}$, we let the normal numerical flux be 0 . We set $\rho=0$ at the ghost points inside the wall. In the Hamilton-Jacobi equation, the numerical boundary values of $\phi$ are obtained by extrapolation from inside the computational domain. In the Eikonal equation, we set $\phi=10^{12}$ at the ghost points.

2. On the boundary of the compact $\mathrm{CBD}$, i.e., $\Gamma_{c}$, we set $\phi=0$ in both the HJ equation and the Eikonal equation. The boundary conditions for $\rho$ inside the CBD are obtained by extrapolation from the grids outside the CBD. To maintain the maximum flow intensity $|\mathbf{F}|=F_{\max }$ on the boundary of the CBD under the congested condition, we set $U(x, y, t)=U_{f}$ inside the CBD.

\subsection{Numerical results}

We now present the numerical results. To verify the convergence of the composed algorithm, we test three grids (grid 1: $140 \times 100$; grid 2: $280 \times 200$; grid 3: $420 \times 300$ ) Note that there are two kinds of convergence to be verified: the convergence of the self-adaptive MSA under each grid, and the convergence among different grids.

Let us consider the first kind of convergence. To demonstrate the effectiveness of our self-adaptive MSA, we plot the discrete points $\left(\lambda_{k}, r_{k}\right), k=2, \cdots, 21$ and the fitted quadratic curve $r^{*}(\lambda)$ under grid 2 in Figure 7 . From the figure, we can see that after the first several iterations using the predetermined step sizes, the self-adaptive MSA is able to find the optimal step size and adjust it within a small range. Figure 8 compares 
the step sizes of our method with the conventional MSA with the step size $\left\{\lambda_{k}=1 / k\right\}$ (Robbins and Monro, 1951) and the self-regulated averaging method (Liu et al., 2009). Figure 9 compares the errors produced by these methods. For the first several iterations, the step size in the self-adaptive MSA decreases faster than in the conventional MSA and the self-regulated averaging method, thus avoiding too large an amplification of the error. After a number of iterations, the step size of the self-adaptive MSA is almost a constant, which can be viewed as the optimal step size. Under this optimal step size, the error continues to decrease at a reasonable speed. However, the step sizes of the other two methods become increasingly smaller as the number of iterations increases, and the error reduction becomes extremely slow. The number of iterations for the MSA with different step size sequences are compared in Table 1. The table shows that the self-adaptive MSA is much faster than the conventional MSA and the self-regulated averaging method.

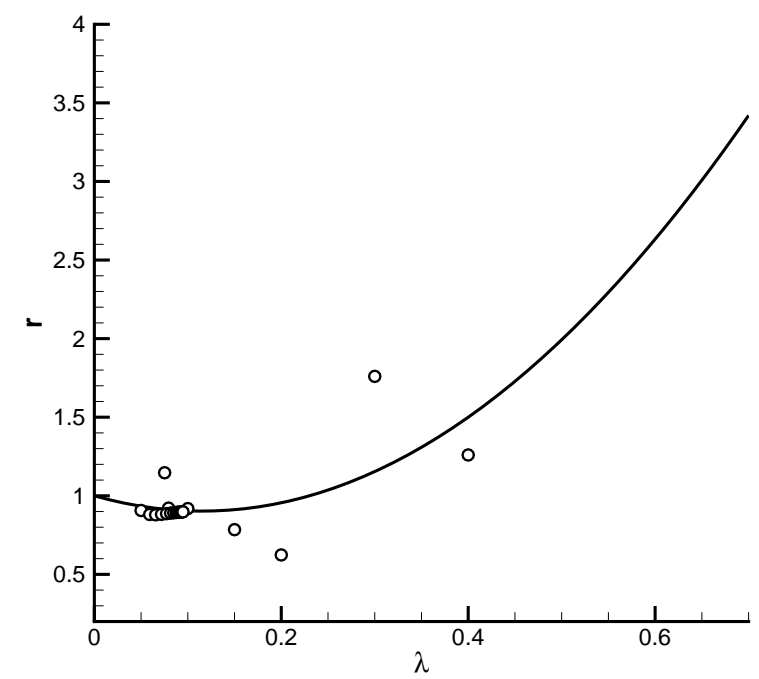

Figure 7: The discrete points and the fitted curve

We now consider the second type of convergence. The density curves at time $t=2$ along different lines are plotted in Figure 10, which shows good convergence among different grids. Grid 2 is adopted for further discussion. 


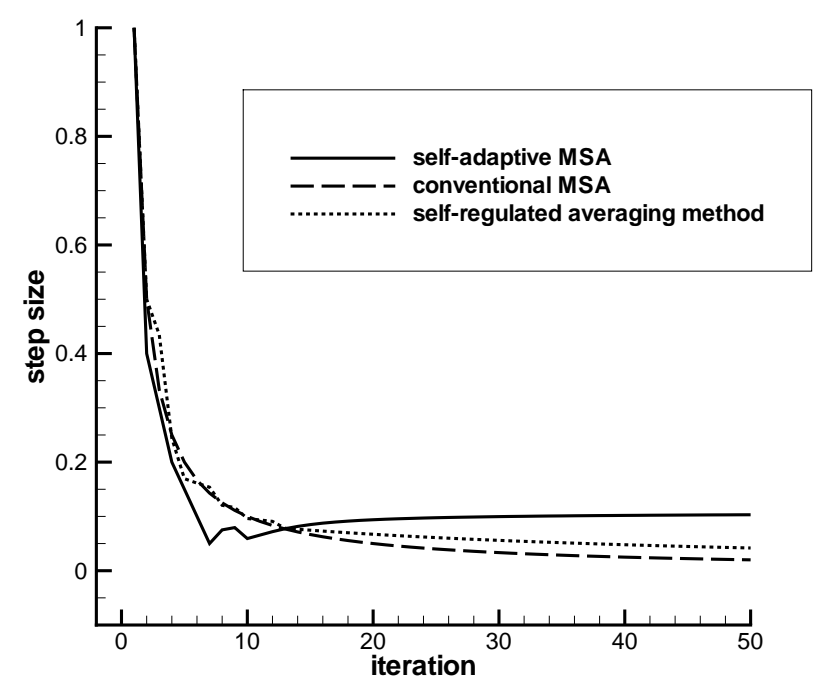

Figure 8: The step sizes of the different methods

Figure 11 shows the temporal and spatial distributions of the density $\rho$ within the modeling region. As shown in Sub-figure 11(a), the density is low at the beginning and the traffic is in the non-congested condition. As the traffic demand grows, the northeast region of the CBD boundary reaches the critical density of $\rho_{c}=500$ before all parts of the CBD boundary become congested, and the density in the eastern region is especially high (see Sub-figure 11(c)). Although the demand starts to decrease from $t=2$, the areas around the $\mathrm{CBD}$ are still in the congested condition at $t=2.5$ due to the limitation on the maximum flow intensity into the CBD (Sub-figure 11(d)). As the demand decreases further, all parts of the city return to the non-congested condition (Sub-figures 11(e) and 11(f)). Finally, all of the travelers have entered the CBD, and there is no traffic in the

\begin{tabular}{|c|c|c|c|}
\hline & Conventional MSA & Self-regulated averaging method & Self-adaptive MSA \\
\hline $140 \times 100$ & $>3000$ & 295 & 87 \\
\hline $280 \times 200$ & $>3000$ & 630 & 180 \\
\hline $420 \times 300$ & $>3000$ & 972 & 235 \\
\hline
\end{tabular}

${ }^{*}$ In the conventional MSA, the $L_{2}$ norm error is still larger than $10^{-2}$ after 3,000 iterations.

Table 1: Iteration numbers for the MSA with different step sizes 


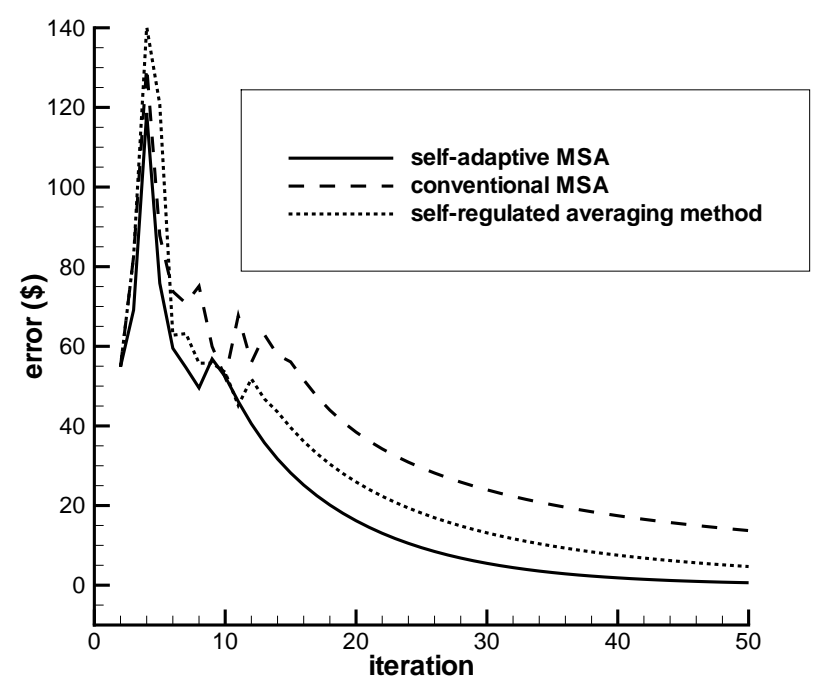

Figure 9: The errors of the different methods

city at $t_{\text {end }}=6$.

Figure 12 illustrates the contours of the total cost potential function $\phi$ at various times. At the beginning of the modeling period, when the density is very low and traffic is in a free-flow state, the cost potential contours are a series of concentric circles located around the CBD. As the traffic demand grows, the total cost potential increases and the contours become more dense. As the spaces between the contours become smaller, the local cost of moving one distance unit increases, as shown in Sub-figure 12(b). In Sub-figures $12(\mathrm{c})$ and $12(\mathrm{~d})$, the density on the eastern side of the CBD is very high and the travel cost increases rapidly, resulting in a denser set of cost potential contours in this region. As the traffic demand decreases, the distributions of the cost potential function return to their former conditions, as shown in Sub-figures 12(e) and 12(f).

Figure 13 shows the plot of the flow vector, which illustrates the results of the path choice. We can see that the travelers pass around the lake if they come from the east. At the beginning and end of the modeling period, as the density is low, vehicles move toward the $\mathrm{CBD}$ in almost straight lines. As the density of the eastern region around 


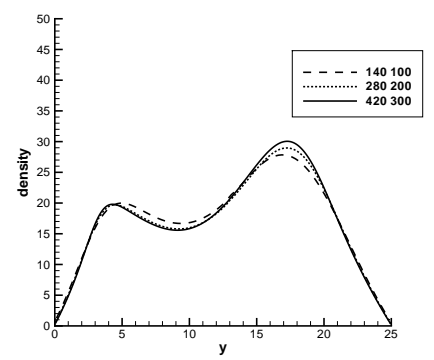

(a) $x=3$

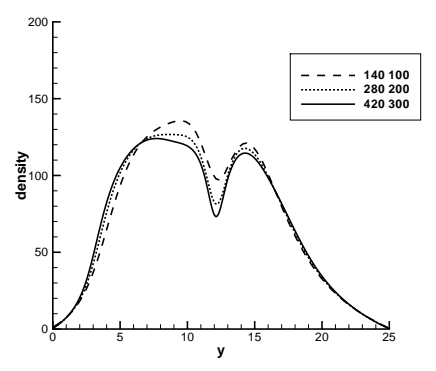

(c) $x=16$

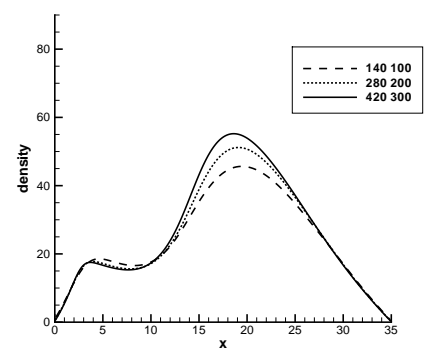

(e) $y=3$

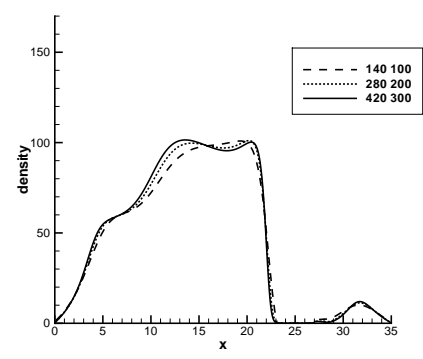

(g) $y=16$

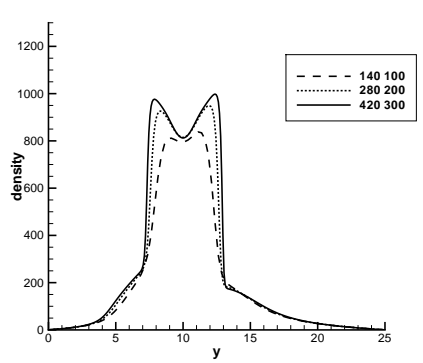

(b) $x=12$

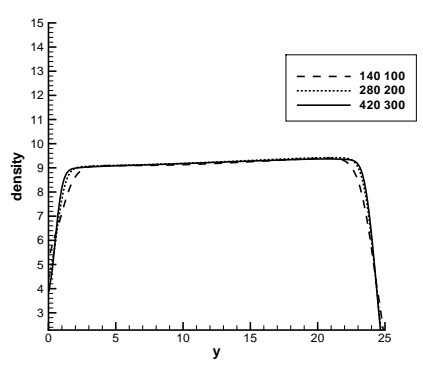

(d) $x=32$

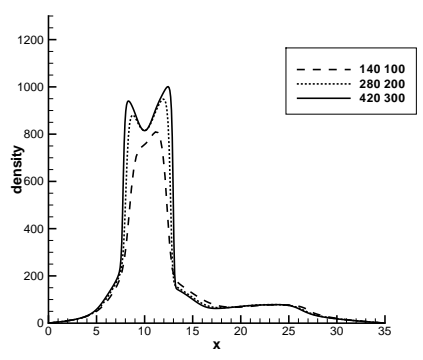

(f) $y=12$

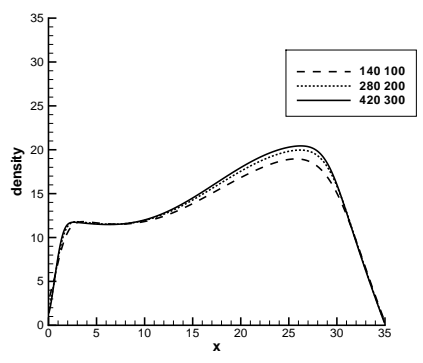

(h) $y=22$

Figure 10: Convergence curves of the density at time $t=2.0$ 


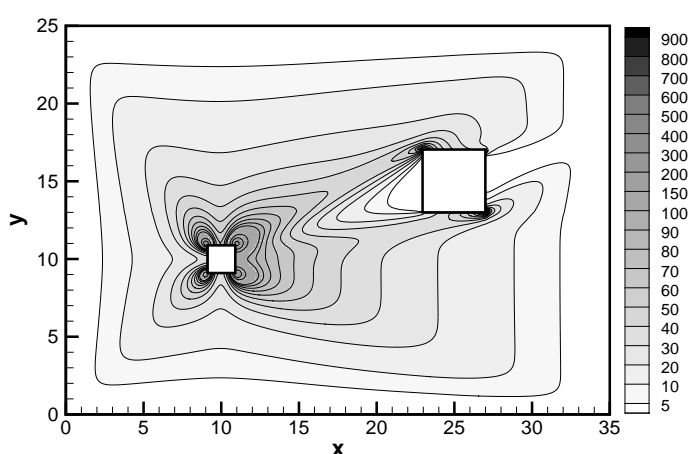

(a) $t=0.5$

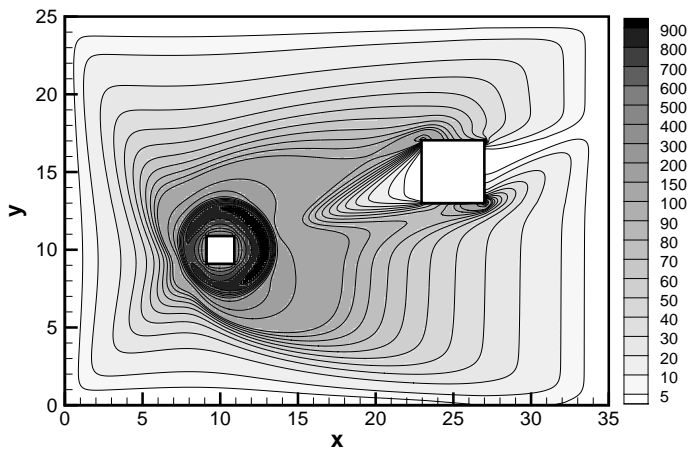

(c) $t=2.0$

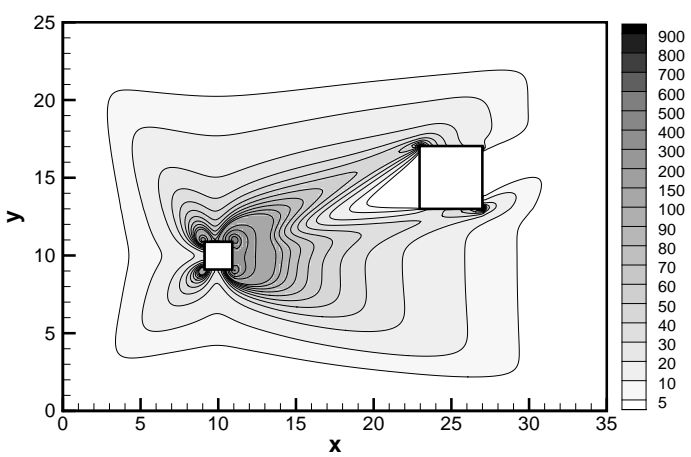

(e) $t=3.0$

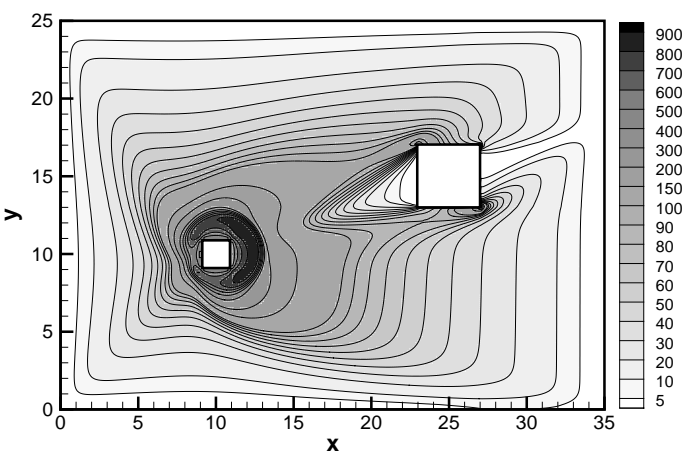

(b) $t=1.5$

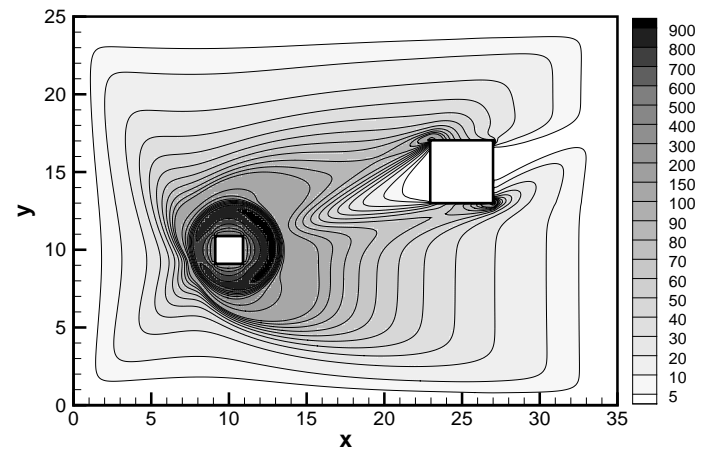

(d) $t=2.5$

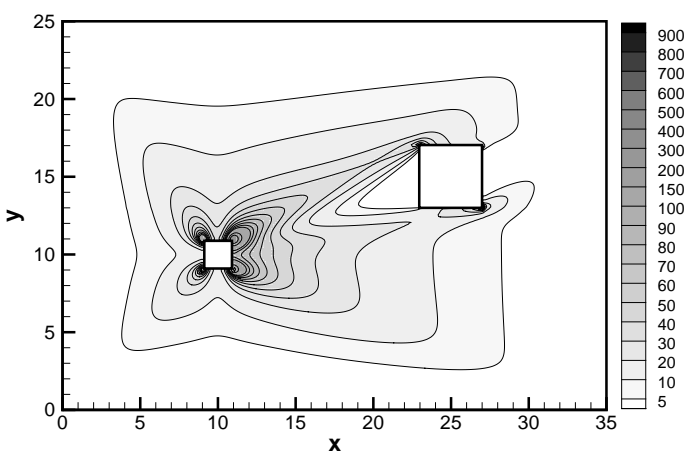

(f) $t=5.0$

Figure 11: Density plot 


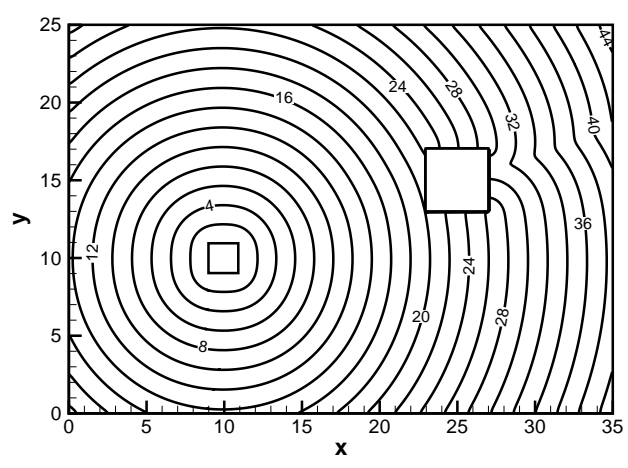

(a) $t=0.5$

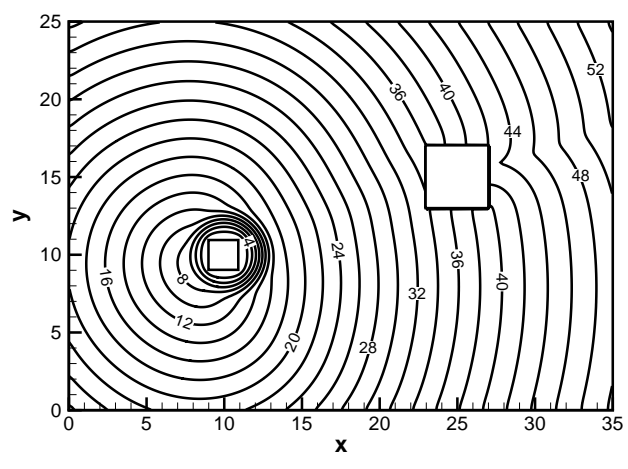

(c) $t=2.0$

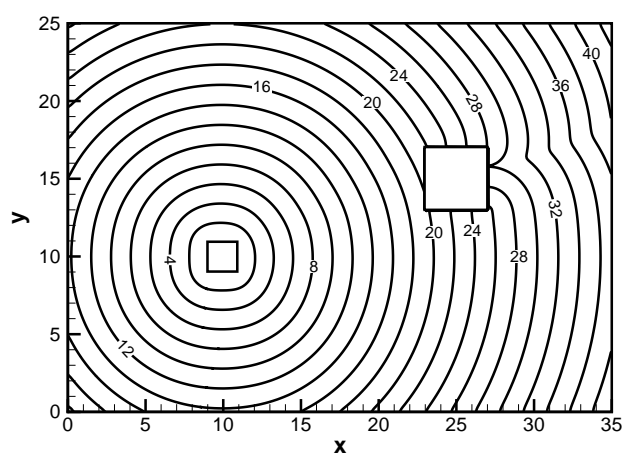

(e) $t=3.0$

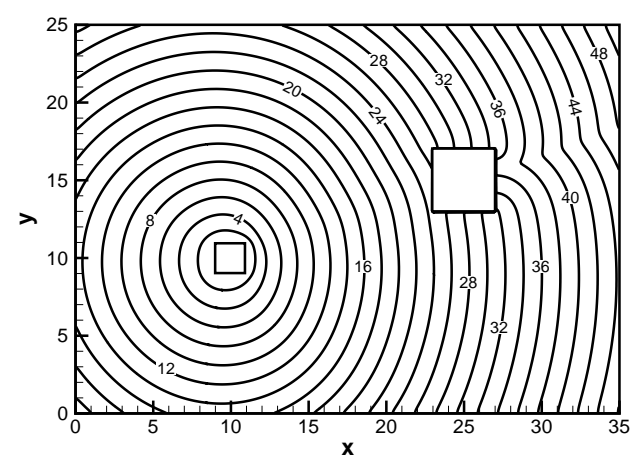

(b) $t=1.0$

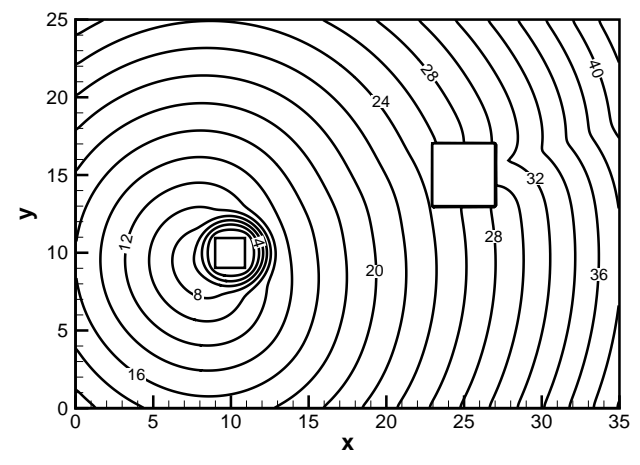

(d) $t=2.5$

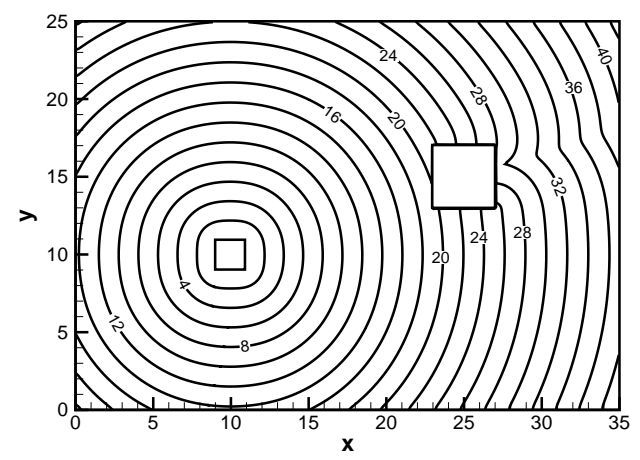

(f) $t=5.0$

Figure 12: Cost potential plot 
the CBD becomes high, vehicles choose a curved trajectory to avoid this region. This demonstrates the capability of the proposed PDUO-C model in modeling how travelers alter their preferred routes in response to dynamic changes in the network conditions to minimize their cost potentials.

Consider the total inflow to the $\mathrm{CBD}$ through $\Gamma_{c}$, defined as

$$
f_{C B D}(t)=\oint_{\Gamma_{c}}(\mathbf{F} \cdot \mathbf{n})(x, y, t) d s
$$

where $\mathbf{n}$ is the unit normal vector pointing toward the CBD, and the total demand over the whole domain is defined as

$$
q_{\Omega}(t)=\iint_{\Omega} q(x, y, t) d x d y
$$

Figure 14 shows the relationship between $f_{C B D}(t)$ and $q_{\Omega}(t)$. The numerical data show that the areas under these two curves are the same, which demonstrates that all of the traffic has entered the CBD by the end of the modeling period. The curve for the total inflow $f_{C B D}(t)$ is always lagging behind the curve for the total demand $q_{\Omega}(t)$. The number of vehicles in the city increases when $q_{\Omega}(t)$ is larger than $f_{C B D}(t)$, and decreases when $f_{C B D}(t)$ is larger than $q_{\Omega}(t)$. As the demand $q_{\Omega}(t)$ grows linearly in $[0 h, 1 h]$ and maintains the maximum value in $[1 h, 2 h]$, the inflow to the $\mathrm{CBD} f_{C B D}(t)$ increases rapidly. When the density around the CBD is greater than the critical density $\rho_{c}$ (Figure 11), $f_{C B D}(t)$ reaches the maximum value and does not increase further, indicating the congested condition. The inflow to the CBD maintains this maximum value, and does not decrease until the density around the CBD decreases below $\rho_{c}$. When the traffic demand starts to decrease from $t=2$, it takes a while to return to the non-congested condition, and the inflow then begins to fall. Note that the total demand maintains a constant $(>0)$ for a while before dropping to 0 , and that the inflow decreases to the same constant after a short delay. The traffic demand falls to 0 after $t=5 h$, and the flow intensity decreases to 0 thereafter. 


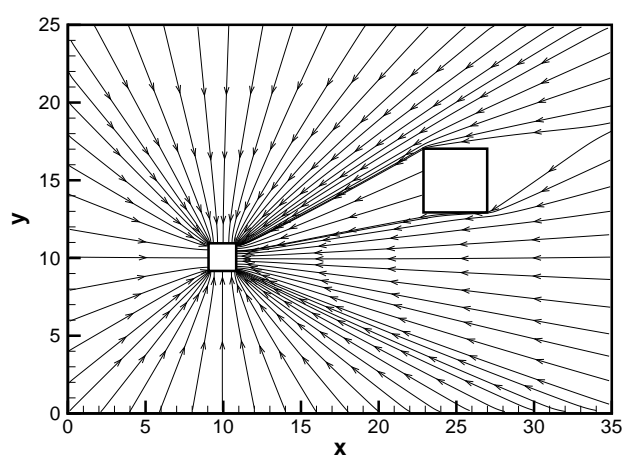

(a) $t=0.5$

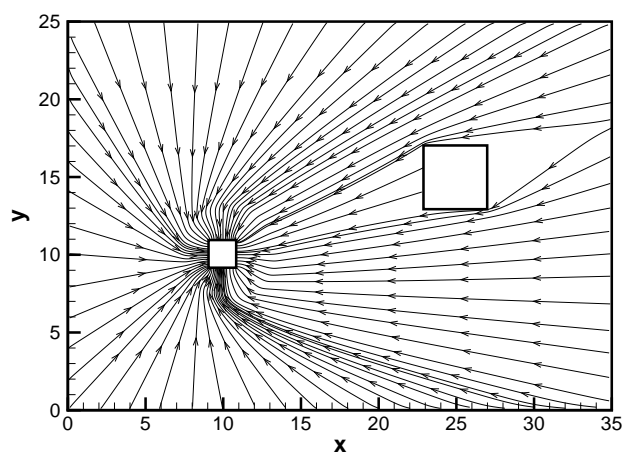

(c) $t=2.0$

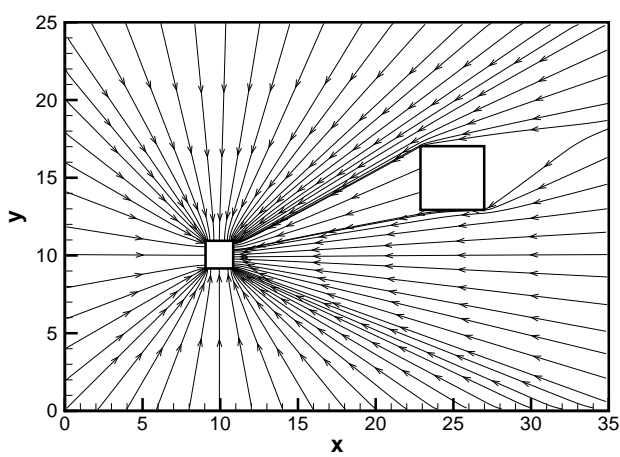

(e) $t=3.0$

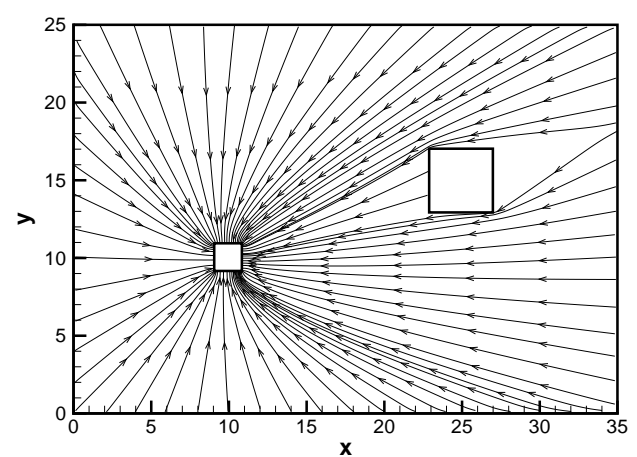

(b) $t=1.0$

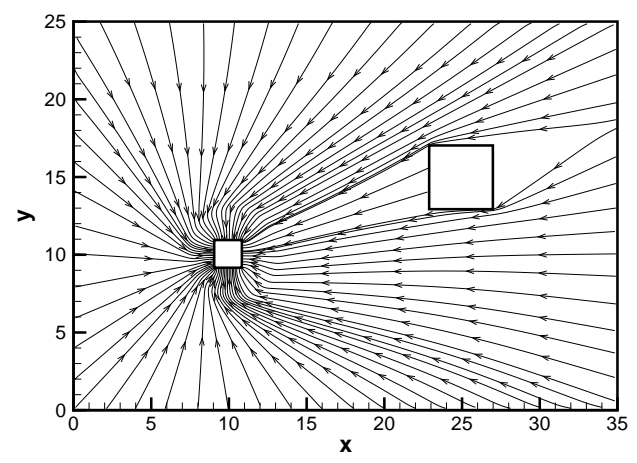

(d) $t=2.5$

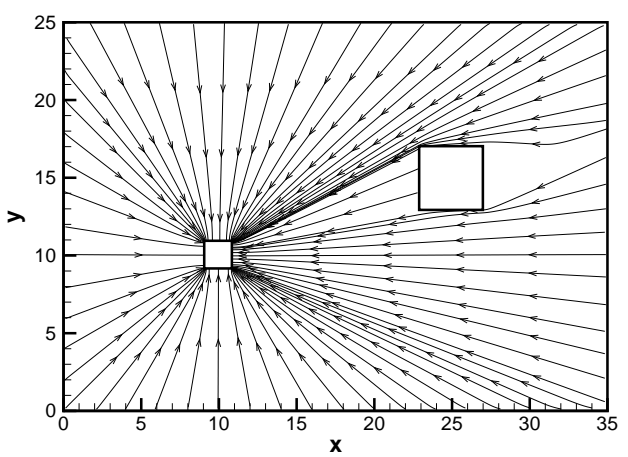

(f) $t=5.0$

Figure 13: Flow vector plot 


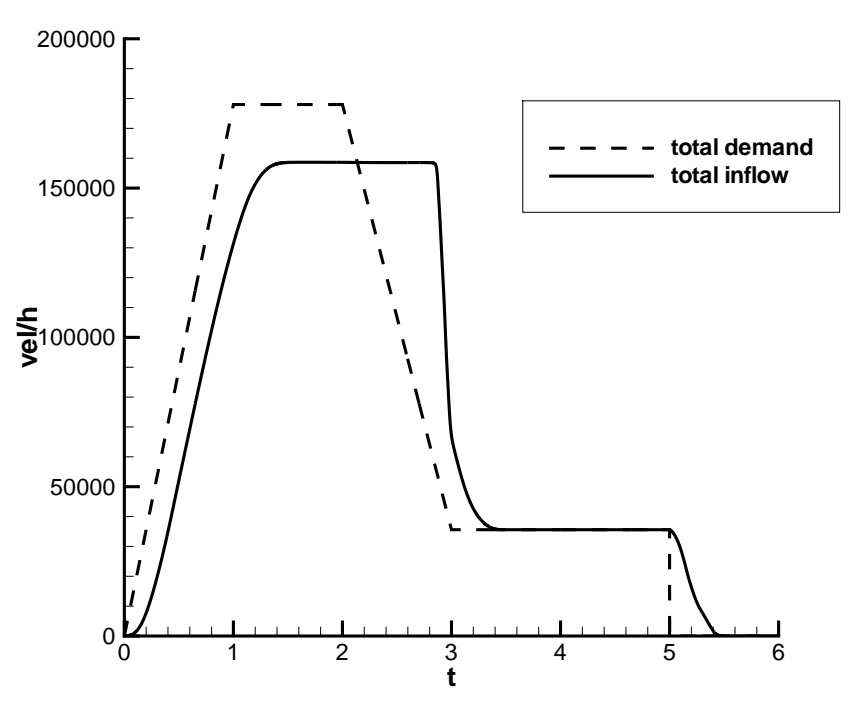

Figure 14: Flow intensity over time

\section{Conclusions}

Due to the inconsistency in the original predictive continuum dynamic user-optimal (PDUO-C) model (Jiang et al., 2011), we revisit this problem and construct an improved path-choice strategy, starting with the special case in which the travel cost is the travel time. The path-choice strategy with a general travel cost is formulated using a different method and is proven to be an extension of the special case. The density of travelers is governed by a conservation law (CL), in which the flow direction is determined by the improved path-choice strategy constructed in this paper. A Hamilton-Jacobi (HJ) equation is derived to compute the travel cost. As the CL and HJ parts must be solved along different directions of time, they cannot be solved together as usual. The model is a fixed-point problem that can be solved using the method of successive averages (MSA). We construct a self-adaptive MSA to solve this fixed-point problem. This method can automatically determine the optimal MSA step size using the least squares method without prior information. The numerical results show that this new self-adaptive MSA is much faster than the conventional methods. The self-adaptive MSA can also be 
adopted for other travel forecasting problems.

It is worth noting that the problem of heterogeneous configurations with different MFDs has recently received much attention. For example, Geroliminis and Sun (2011) identified the spatial distribution of vehicle density in the network as one of the key components affecting the scatter of an MFD and its shape. While examining the existence of MFDs and the feasibility of simple control strategies to improve network performance in heterogeneously congested networks, Ji and Geroliminis (2012) recently addressed the question of how to decompose road networks into subnetworks so that each subnetwork has a well-defined MFD. The dynamic continuum model presented in this paper offers an alternative approach to the study of this interesting problem. The speed-density relationship in Equation (2) is location dependent, which means that the MFD parameters can vary geographically in representing heterogeneous configurations of dense road networks. Therefore, the dynamic continuum model can be applied to the case of heterogeneous regions effectively.

The formulation in this paper is only for the region with a single CBD. In the case of multiple CBDs, the flows would interact in the city, and thus the model would become more complicated. To study this problem, we have to understand the directional effects of how different copies of flows, one for each CBD, interact when they cross each other at the city domain, analogous to previous work on pedestrian flows (Wong et al., 2010; Xiong et al., 2011). This would make for an interesting future study.

In this paper, we develop a numerical scheme based on a rectangular grid. It would be useful to extend the numerical scheme based on a triangular grid system, which is more flexible for tackling complicated geometry. Extensions such as a polycentric city with multiple CBDs and a combined departure time and assignment could be developed in future studies. 


\section{Acknowledgements}

The work described in this paper was supported by grants from the Research Grants

Council of the Hong Kong Special Administrative Region, China (HKU 7184/10E), a National Research Foundation of Korea grant from the Korean government (MEST) (NRF-2010-0029446), US ARO grant W911NF-11-1-0091, US NSF grant DMS-1112700 and NSFC grants 11071234 and 91024025.

\section{References}

Bar-Gera, H., Boyce, D., 2006. Solving a non-convex combined travel forecasting model by the method of successive averages with constant step sizes. Transportation Research Part B 40 (5), 351-367.

Blumenfeld, D.E., 1977. Modeling the joint distribution of home and workplace location in a city. Transportation Science 11 (4), 307-377.

Blumenfeld, D.E., Weiss, G.H., 1970. Routing in a circular city with two ring roads. Transportation Research 4 (2), 235-242.

Boyce, D.E., Ran, B., Leblanc, L.J., 1993. Solving an instantaneous dynamic useroptimal route choice model. Transportation Science 29 (2), 128-142.

Buckley, D.J., 1979. Traffic assignment in a two-dimensional continuous representation of a traffic network with flow-dependent speeds. Transportation Research Part B 13 (2), 167-179.

Chow, A.H.F., 2009. Dynamic system optimal traffic assignment - a state-dependent control theoretic approach. Transportmetrica 5 (2), 85-106.

Dafermos, S.C., 1980. Continuum modeling of transportation networks. Transportation Research Part B 14 (3), 295-301. 
Daganzo, C.F., Gayah, V.V., Gonzales, E.J., 2011. Macroscopic relations of urban traffic variables: bifurcations, multivaluedness and instability. Transportation Research Part B $45(1), 278-288$.

Daganzo, C.F., Geroliminis, N., 2008. An analytical approximation for the macroscopic fundamental diagram of urban traffic. Transportation Research Part B 42 (9), 771-781.

Gayah, V.V., Daganzo, C.F., 2011. Clockwise hysteresis loops in the macroscopic fundamental diagram: an effect of network instability. Transportation Research Part B 45 (4), 643-655.

Geroliminis, N., Boyaci, B., 2012. The effect of variability of urban systems characteristics in the network capacity. Transportation Research Part B 46 (10), 1607-1623.

Geroliminis, N., Daganzo, C.F., 2008. Existence of urban-scale macroscopic fundamental diagrams: some experimental findings. Transportation Research Part B 42 (9), 759770.

Geroliminis, N., Sun, J., 2011. Properties of a well-defined macroscopic fundamental diagram for urban traffic. Transportation Research Part B 45 (3), 605-617.

Ho, H.W., Wong, S.C., Loo, B.P.Y., 2006. Combined distribution and assignment model for a continuum traffic equilibrium problem with multiple user classes. Transportation Research Part B 40 (8), 633-650.

Ho, H.W., Wong, S.C., Sumalee, A., 2013. A congestion-pricing problem with a polycentric region and multi-class users: a continuum modelling approach. Transportmetrica, DOI:10.1080/18128602.2011.621652.

Hoogendoorn, S.P., Bovy, P.H.L., 2004. Dynamic user-optimal assignment in continuous time and space. Transportation Research Part B 38 (7), 571-592. 
Huang, L., Wong, S.C., Zhang, M.P., Shu, C.W., Lam, W.H.K., 2009. Revisiting Hughes' dynamic continuum model for pedestrian flow and the development of an efficient solution algorithm. Transportation Research Part B 43 (1), 127-141.

Ji, Y., Geroliminis, N., 2012. On the spatial partitioning of urban transportation networks. Transportation Research Part B 46 (10), 1639-1656.

Jiang, Y.Q., Wong, S.C., Ho, H.W., Zhang, P., Liu, R.X., Sumalee, A., 2011. A dynamic traffic assignment model for a continuum transportation system. Transportation Research Part B 45 (2), 343-363.

Kuwahara, M., Akamatsu, T., 2001. Dynamic user optimal assignment with physical queue for a many-to-many OD pattern. Transportation Research Part B 35 (5), 461479.

Lam, T.N., Newell, G.F., 1967. Flow dependent traffic assignment on a circular city. Transportation Science 1 (4), 318-361.

Lee, D.H., 2002. Urban and Regional Transportation Modeling: Essays in Honor of David Boyce. Edward Elgar Publishing Inc., Northampton, USA.

Liu, H.X., He, X.Z., He, B.S., 2009. Method of successive weighted averages (MSWA) and self-regulated averaging schemes for solving stochastic user equilibrium problem. Networks and Spatial Economics 9 (4), 485-503.

Lo, H.K., Szeto, W.Y., 2002. A cell-based variational inequality formulation of the dynamic user optimal assignment problem. Transportation Research Part B 36 (5), 421443.

Lo, H.K., Szeto, W.Y., 2005. Road pricing modeling for hyper-congestion. Transportation Research Part A 39 (7-9), 705-722. 
Nagurney, A., Zhang, D., 1996. Projected Dynamical Systems and Variational Inequalities with Applications. Kluwer, Boston, Massachusetts.

Polyak, B.T., 1990. New method of stochastic approximation type. Automation and Remote Control 51 (7), 937-946.

Robbins, H., Monro, S., 1951. A stochastic approximation method. Annals of Mathematical Statistics 22 (3), 400-407.

Szeto, W.Y., Lo, H.K., 2004. A cell-based simultaneous route and departure time choice model with elastic demand. Transportation Research Part B 38 (7), 593-612.

Taguchi, A., Iri, M., 1982. Continuum approximation to dense networks and its application to the analysis of urban road networks. Mathematical Programming Study 20, $178-217$.

Tong, C.O., Wong, S.C., 1997. The advantages of a high density, mixed land use, linear urban development. Transportation 24 (33), 295-307.

Tong, C.O., Wong, S.C., 2000. A predictive dynamic traffic assignment model in congested capacity-constrained road networks. Transportation Research Part B 34 (8), $625-644$

Vaughan, R.J., 1985. A continuous analysis of the role of transportation and crowding costs in determining trip distribution and location in a linear city. Transportation Research Part A 19 (2), 89-107.

Vaughan, R.J., 1987. Urban Spatial Traffic Patterns. Pion, London, U.K.

Williams, H.C.W.L., Ortuzar, J.D., 1976. Some generalizations and applications of the velocity field concept: Trip patterns in idealized cities. Transportation Research 10 (2), 65-73. 
Wong, S.C., 1998. Multi-commodity traffic assignment by continuum approximation of network flow with variable demand. Transportation Research Part B 32 (8), 567-581.

Wong, S.C., Lee, C.K., Tong, C.O., 1998. Finite element solution for the continuum traffic equilibrium problems. International Journal for Numerical Methods in Engineering $43(7), 1253-1273$.

Wong, S.C., Leung, W.L., Chan, S.H., Lam, W.H.K., Yung, N.H.C., Liu, C.Y. and Zhang, P., 2010. Bidirectional pedestrian stream model with oblique intersecting angle. ASCE Journal of Transportation Engineering 136, 234-242.

Xiong, T., Zhang, M., Shu, C.W., Wong, S.C. and Zhang, P., 2011. High-order computational scheme for a dynamic continuum model for bi-directional pedestrian flows. Computer-Aided Civil and Infrastructure Engineering 26, 298-310.

Yin, J., Wong, S.C., Sze, N.N., Ho, H.W., 2013. A continuum model for housing allocation and transportation emission problems in a polycentric city. International Journal of Sustainable Transportation 7, 275-298.

Zhang, X., Huang, H.J., Zhang, H.M., 2008. Integrated daily commuting patterns and optimal road tolls and parking fees in a linear city. Transportation Research Part B $42(1), 38-56$.

Zhao, H.K., 2005. A fast sweeping method for Eikonal equations. Mathematics of Computation $74(250), 603-627$.

Zitron, N.R., 1978. A critical condition for the cost density in the circular city model. Journal of Optimization Theory and Application 24 (3), 507-512. 


\section{Appendix A. The detailed proof of Theorem 1}

Theorem 1: If we choose the speed vector $\mathbf{v}$ such that the resulting $\nabla z$ is always parallel to $\mathbf{v}$ and has the same direction, then the dynamic predictive user equilibrium in terms of total travel time to the CBD is satisfied.

Proof: If $\nabla z \| \mathbf{v}$, then we have

$$
\nabla z \cdot \mathbf{v}=|\nabla z||\mathbf{v}|=|\nabla z| U
$$

where $U=\sqrt{u^{2}+v^{2}}$ is the given isotropic speed in the time-space domain. Using Equation (13), we can obtain

$$
|\nabla z| U=1
$$

and hence

$$
\left|\nabla z\left(x, y, t_{D}\right)\right|=1 / U\left(x, y, z\left(x, y, t_{D}\right)\right)
$$

Next, consider Figure A.1, in which the larger circle has a radius $\Delta t U$, which is the boundary of distance movement in $\Delta t$ time, as the isotropic speed is $U$. Here, we assume that $\Delta t$ is small enough such that $z\left(x, y, t_{D}\right)$ can be treated as a linear function of $x$ and $y$ in such a small area. The dotted curve represents the "used" path from $(x, y)$ to the CBD based on the parallel condition $\nabla z \| \mathbf{v}$. The total travel time along this "used" path becomes the difference between the departure time $z\left(x, y, t_{D}\right)$ from $\left(x, y, z\left(x, y, t_{D}\right)\right)$ and the arrival time $t_{D}$ at the CBD, and thus

$$
\phi\left(x, y, z\left(x, y, t_{D}\right)\right)=t_{D}-z\left(x, y, t_{D}\right) .
$$

We need to show that for any path from $\left(x, y, z\left(x, y, t_{D}\right)\right)$ that deviates from this "used" path (3D trajectory), the arrival time will be no earlier than $t_{D}$, i.e., the total travel time will be no less than $\phi\left(x, y, z\left(x, y, t_{D}\right)\right)$. We can prove this using the following two steps.

Step 1. Let us first consider an "unused" path that deviates from the "used" path only in the first $\Delta t$ time and then reverts back to the "used" path. As shown in Figure A.1, we 


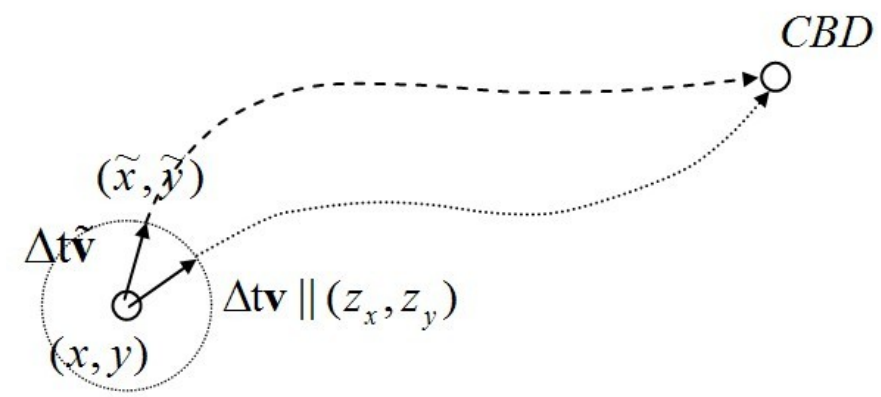

Figure A.1: The "used" and "unused" paths

consider the path that moves away from the starting point with a vector $\Delta t \tilde{\mathbf{v}}=\Delta t(\tilde{u}, \tilde{v})$, where $|\tilde{\mathbf{v}}|=U$ is the isotropic speed, followed by the dashed curve that represents the 3D "used" trajectory from $(\tilde{x}, \tilde{y})$ to the CBD, where $(\tilde{x}, \tilde{y})$ is the position at which the vehicle arrives at the dotted circle boundary in $\Delta t$ time with the vector $\Delta t \tilde{\mathbf{v}}$. As $\tilde{\mathbf{v}}=(\tilde{u}, \tilde{v})$ is not parallel with $\nabla z=\left(z_{x}, z_{y}\right)$, by defining the angle between $\mathbf{v}$ and $\tilde{\mathbf{v}}$ as $\theta$, we can show that the change in $z$ at point $\left(x, y, z\left(x, y, t_{D}\right)\right.$ along the movement vector $\Delta t \tilde{\mathbf{v}}$ is

$$
\begin{aligned}
& z\left(\tilde{x}, \tilde{y}, t_{D}\right)-z\left(x, y, t_{D}\right) \\
= & \nabla z \cdot \Delta t \tilde{\mathbf{v}} \\
= & |\nabla z||\tilde{\mathbf{v}}| \cos \theta \Delta t=|\nabla z| U \cos \theta \Delta t \\
\leq & |\nabla z| U \Delta t .
\end{aligned}
$$

Here, the first equal sign comes from the assumption that $z\left(x, y, t_{D}\right)$ is a linear function of $x$ and $y$ in this small area. Even without this assumption, as $\Delta t$ is small enough, the nonlinear item in the Taylor expansion is the high order infinitesimal, which makes no difference to our discussion and can be neglected. Using Equation (A.3 ), we have

$$
z\left(\tilde{x}, \tilde{y}, t_{D}\right)-z\left(x, y, t_{D}\right) \leq \frac{\Delta t}{U} U=\Delta t
$$

i.e.,

$$
z\left(x, y, t_{D}\right)+\Delta t \geq z\left(\tilde{x}, \tilde{y}, t_{D}\right)
$$

This means that the arrival time at $(\tilde{x}, \tilde{y})$ along this "unused" path, i.e., $z\left(x, y, t_{D}\right)+\Delta t$, 
will not be earlier than the departure time from $(\tilde{x}, \tilde{y})$ that is required to reach the CBD at time $t_{D}$, i.e., $z\left(\tilde{x}, \tilde{y}, t_{D}\right)$. The central question is whether a vehicle that departs from $(\tilde{x}, \tilde{y})$ at a later time could arrive at the CBD earlier than $t_{D}$, even if the vehicle follows the "used" path for the reminder of the journey. We can show that this is not possible by contradiction.

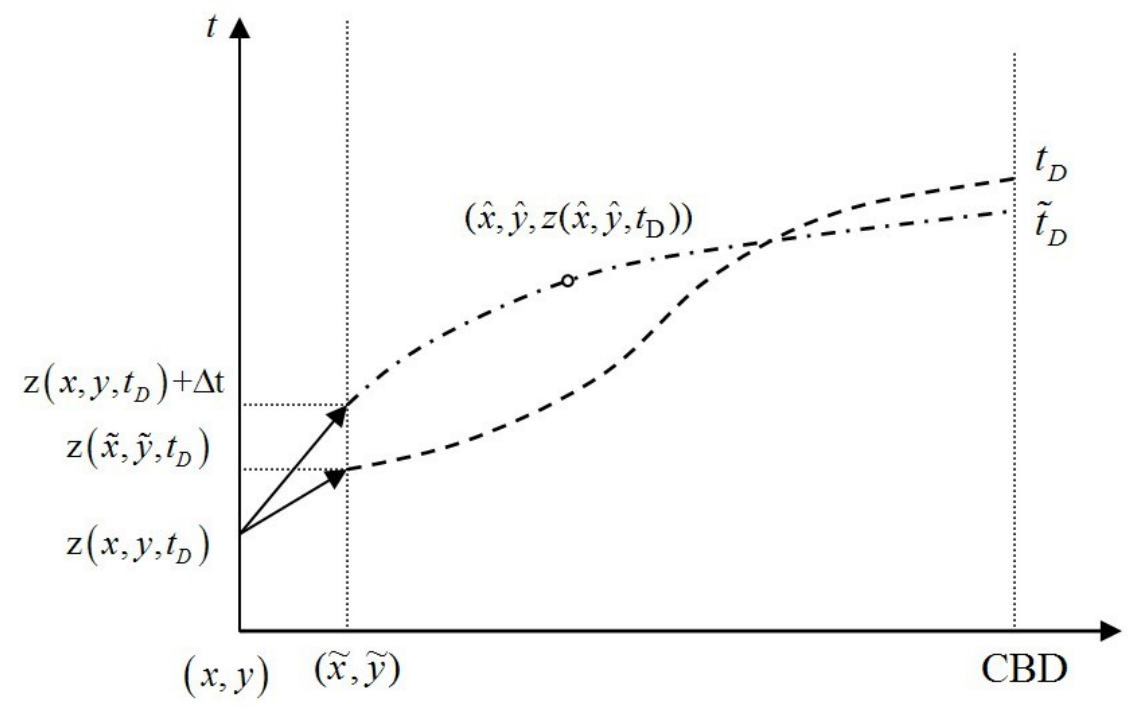

Figure A.2: Two "used" paths from $(\tilde{x}, \tilde{y})$ to the CBD

To illustrate this, consider Figure A.2. Suppose that it is possible to arrive at the CBD at an earlier time $\tilde{t}_{D}$, as shown by the dotted-dashed line, even if the vehicle departs later. As $z\left(x, y, t_{D}\right)$ is a closed surface emanating from $\left(C B D, t_{D}\right)$, the dotted-dashed curve that leaves $(\tilde{x}, \tilde{y})$ at time $z\left(x, y, t_{D}\right)+\Delta t \geq z\left(\tilde{x}, \tilde{y}, t_{D}\right)$ and arrives at the CBD at $\tilde{t}_{D}<t_{D}$ must cut through the closed surface $z\left(x, y, t_{D}\right)$ somewhere at $\left(\hat{x}, \hat{y}, z\left(\hat{x}, \hat{y}, t_{D}\right)\right)$. However, by definition, if a vehicle departs from this time-space point, it can reach the CBD at both $t_{D}$ and $\tilde{t}_{D}$, which is impossible.

Therefore, if the vehicle moves in any direction other than $\mathbf{v}=(u, v)$ in the first $\Delta t$ time, it will not arrive at the CBD earlier than $t_{D}$, resulting in a total travel time of no less than $\phi\left(x, y, z\left(x, y, t_{D}\right)\right)$ along the used path.

Step 2. Now let us consider the more general case. In step 1, if a vehicle moves along 
$\tilde{\mathbf{v}}=(\tilde{u}, \tilde{v})$ in the first $\Delta t$ time, it will arrive at the CBD at time $\tilde{t}_{D} \geq t_{D}$. However, if it does not revert back to the "used" path in the next step and continues to move in a direction other than the speed vector, then by the same token it will arrive even later at $\tilde{\tilde{t}}_{D} \geq \tilde{t}_{D} \geq t_{D}$. Therefore, for any "unused" path, the total travel time must be no less than that of the "used" path.

\section{Appendix B. The detailed proof of Theorem 2}

Theorem 2: If $(u, v) \|\left(-\phi_{x},-\phi_{y}\right)$, then the predictive dynamic user equilibrium principle is satisfied.

Proof: Recall from the definition in the problem description section that $\phi(x, y, t)$ is the total travel cost incurred by a traveler who departs from location $(x, y)$ at time $t$ to travel to the CBD using the constructed path-choice strategy. This requires traveling along the path that satisfies $(u, v) \|\left(-\phi_{x},-\phi_{y}\right)$, i.e., the "used" path. We need to show that for any "unused" path from $O\left(x_{0}, y_{0}, t_{0}\right)$ that deviates from the "used" path, the total cost must be no less than $\phi\left(x_{0}, y_{0}, t_{0}\right)$. We can prove this using the following two steps.

Step 1. We first consider the "unused" path that deviates from the "used" path only at the initial $\Delta t$ time and reverts back to the "used" path thereafter. Again, we assume that $\Delta t$ is small enough such that we can neglect the high order infinitesimal in the Taylor expansion. Consider Figure B.1, in which the circle has a radius $\Delta t U$, i.e., the boundary of moving $\Delta t$ time from location $O\left(x_{0}, y_{0}, t_{0}\right) . A(x, y)$ is the position at which the vehicle arrives along the "used" path with a vector $\Delta t(u, v)$ based on the parallel condition $(u, v) \|\left(-\phi_{x},-\phi_{y}\right)$, where $U=\sqrt{u^{2}+v^{2}}$ is the given isotropic speed in the time-space domain. $\tilde{A}(\tilde{x}, \tilde{y})$ is the position at which the vehicle arrives along the "unused" path with a vector $\Delta t(\tilde{u}, \tilde{v})$. The arrival times at $A$ and $\tilde{A}$ are obviously both $t_{0}+\Delta t$. 


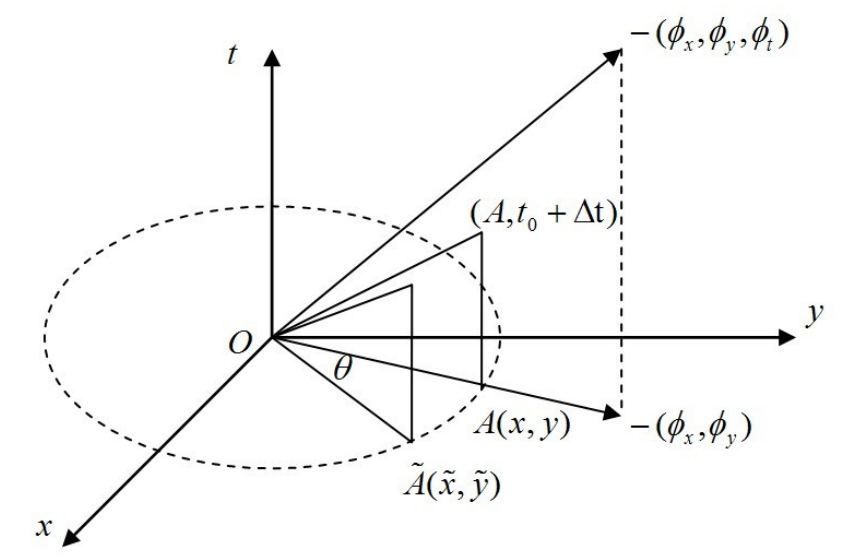

Figure B.1: The "used" and "unused" paths

We can show that the change in $\phi$ along the "used" path is

$$
\begin{aligned}
& \phi\left(x, y, t_{0}+\Delta t\right)-\phi\left(x_{0}, y_{0}, t_{0}\right) \\
= & \left(\phi_{x}, \phi_{y}, \phi_{t}\right) \cdot(u, v, 1) \Delta t \\
= & \Delta t\left[\left(\phi_{x}, \phi_{y}\right) \cdot(u, v)+\phi_{t}\right] \\
= & \Delta t\left(-|\nabla \phi| U+\phi_{t}\right) .
\end{aligned}
$$

The change in $\phi$ along the "unused" path is

$$
\begin{aligned}
& \phi\left(\tilde{x}, \tilde{y}, t_{0}+\Delta t\right)-\phi\left(x_{0}, y_{0}, t_{0}\right) \\
= & \left(\phi_{x}, \phi_{y}, \phi_{t}\right) \cdot(\tilde{u}, \tilde{v}, 1) \Delta t \\
= & \Delta t\left[\left(\phi_{x}, \phi_{y}\right) \cdot(\tilde{u}, \tilde{v})+\phi_{t}\right] \\
= & \Delta t\left(-|\nabla \phi| U \cos \theta+\phi_{t}\right) \\
\geq & \Delta t\left(-|\nabla \phi| U+\phi_{t}\right) .
\end{aligned}
$$

Hence, we have

$$
\begin{gathered}
\phi\left(\tilde{x}, \tilde{y}, t_{0}+\Delta t\right)-\phi\left(x_{0}, y_{0}, t_{0}\right) \geq \phi\left(x, y, t_{0}+\Delta t\right)-\phi\left(x_{0}, y_{0}, t_{0}\right), \\
\phi\left(\tilde{x}, \tilde{y}, t_{0}+\Delta t\right) \geq \phi\left(x, y, t_{0}+\Delta t\right) .
\end{gathered}
$$


Note that the travel costs from point $O\left(x_{0}, y_{0}, t_{0}\right)$ to $A$ and $\tilde{A}$ are both $\Delta t U c(x, y, t)$. We clearly have

$$
\phi\left(x_{0}, y_{0}, t_{0}\right)=\Delta t U c\left(x_{0}, y_{0}, t_{0}\right)+\phi\left(x, y, t_{0}+\Delta t\right)
$$

and the cost along the "unused" path that deviates from the "used" path only in the first $\Delta t$ period is

$$
\tilde{\phi}=\Delta t U c\left(x_{0}, y_{0}, t_{0}\right)+\phi\left(\tilde{x}, \tilde{y}, t_{0}+\Delta t\right)
$$

As $\phi\left(\tilde{x}, \tilde{y}, t_{0}+\Delta t\right) \geq \phi\left(x, y, t_{0}+\Delta t\right)$, the total cost along the "unused" path must be no less than $\phi\left(x_{0}, y_{0}, t_{0}\right)$.

Step 2. In the aforementioned case, if a vehicle moves along $(\tilde{u}, \tilde{v})$ in the first $\Delta t$ period, it will arrive at the $\mathrm{CBD}$ with the cost $\tilde{\phi} \geq \phi\left(x_{0}, y_{0}, t_{0}\right)$. However, if it does not revert back to the "used" path in the next step and continues to move in a direction other than that of the speed vector, then by the same token it will arrive at the CBD with a cost $\tilde{\tilde{\phi}} \geq \tilde{\phi} \geq \phi\left(x_{0}, y_{0}, t_{0}\right)$. Therefore, for any "unused" path, the total cost must be no less than that of the "used" path.

\section{Appendix C. The detailed proof of Theorem 3}

Theorem 3: In the case that the cost $\phi(x, y, t)$ is the travel time in the domain, we have $\phi_{t} \geq-1$.

Proof: The proof is similar to the proof of the dynamic user equilibrium principle in Theorem 1. As shown in Figure C.1, suppose a vehicle can arrive at the CBD at time $t_{D}$ along the "used" path from point $\left(x, y, t_{0}\right)$, and can arrive at the CBD at time $\hat{t}_{D}$ along the "used" path from $\left(x, y, t_{0}+\Delta t\right)$. From Section 4.1, we know that if a vehicle departs from $(x, y)$ at a later time, it will arrive at the CBD later, so we have

$$
\begin{cases}\hat{t}_{D} \geq t_{D}, & \text { if } \Delta t \geq 0 \\ \hat{t}_{D} \leq t_{D}, & \text { if } \Delta t<0\end{cases}
$$




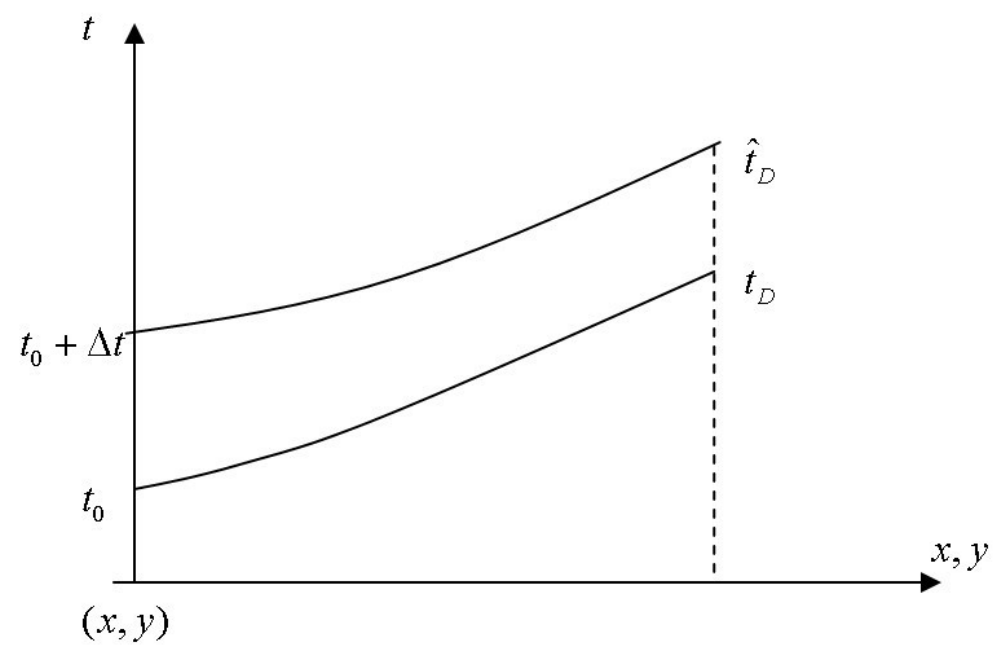

Figure C.1: Two "used" paths

Now we can estimate the value of $\phi_{t}$ as follows:

$$
\begin{aligned}
\phi_{t} & =\lim _{\Delta t \rightarrow 0} \frac{\phi\left(x, y, t_{0}+\Delta t\right)-\phi\left(x, y, t_{0}\right)}{\Delta t} \\
& =\lim _{\Delta t \rightarrow 0} \frac{\left[\hat{t}_{D}-\left(t_{0}+\Delta t\right)\right]-\left[t_{D}-t_{0}\right]}{\Delta t} \\
& =\lim _{\Delta t \rightarrow 0} \frac{\hat{t}_{D}-t_{D}}{\Delta t}-1 .
\end{aligned}
$$

Using Equation (C.1), we get $\phi_{t} \geq-1$.

\section{Appendix D. Fast sweeping method to solve the Eikonal equation}

Before solving the HJ equation, we need the initial value for $\phi_{0}(x, y)$ to start the numerical computation, which is computed using an Eikonal equation:

$$
\begin{cases}\left|\nabla \phi_{0}(x, y)\right|=c\left(x, y, t_{\text {end }}\right), & \forall(x, y) \in \Omega, \\ \phi_{0}(x, y)=\phi_{C B D}, & \forall(x, y) \in \Gamma_{c},\end{cases}
$$

where $c(x, y, t)=90\left(\frac{1}{U}+10^{-8} \rho^{2}\right)$ and $U(x, y, t)=U_{f} e^{-\beta \rho^{2}}$.

The Eikonal equation is in fact a special steady-state Hamilton-Jacobi equation. We use the first-order Godunov fast sweeping method (Zhao, 2005) to solve it. We first 
assign the exact boundary values $\phi_{0}(x, y)=\phi_{C B D}$ to $\Gamma_{c}$. Large values such as $10^{6}$ are assigned as the initial guess values at all other grid points. The following Gauss-Seidel iterations with four alternating direction sweepings are performed:

$$
\begin{aligned}
& \text { (1) } i=1: N_{x}, j=1: N_{y} ; \quad \text { (2) } i=N_{x}: 1, j=1: N_{y} \\
& \text { (3) } i=N_{x}: 1, j=N_{y}: 1 ; \quad \text { (4) } i=1: N_{x}, j=N_{y}: 1,
\end{aligned}
$$

where $i$ and $j$ are the grid indexes in x and y, respectively. $N_{x}$ and $N_{y}$ are the number of grid points in $\mathrm{x}$ and $\mathrm{y}$, respectively. When we loop to the point $(i, j)$, the following formula is used to update the solution:

$$
\phi_{i, j}^{\text {new }}= \begin{cases}\min \left(\phi_{i, j}^{x \min }, \phi_{i, j}^{y \min }\right)+c_{i, j} h, & \text { if }\left|\phi_{i, j}^{x \min }-\phi_{i, j}^{y \min }\right| \geq c_{i, j} h, \\ \frac{\phi_{i, j}^{x \min }+\phi_{i, j}^{y \min }+\left(2 c_{i, j}^{2} h^{2}-\left(\phi_{i, j}^{x \min }-\phi_{i, j}^{y \min }\right)^{2}\right)^{\frac{1}{2}}}{2}, & \text { otherwise, }\end{cases}
$$

where $c_{i, j}=c\left(x_{i}, y_{j}, t_{e n d}\right), \phi_{i, j}^{x \min }$ and $\phi_{i, j}^{y \min }$ are defined as

$$
\left\{\begin{array}{l}
\phi_{i, j}^{x \min }=\min \left(\phi_{i-1, j}, \phi_{i+1, j}\right) \\
\phi_{i, j}^{y \min }=\min \left(\phi_{i, j-1}, \phi_{i, j+1}\right)
\end{array}\right.
$$

Convergence is declared if

$$
\left\|\phi^{\text {new }}-\phi^{\text {old }}\right\| \leq \delta
$$

where $\delta$ is a given convergence threshold value. We use $\delta=10^{-9}$ and $L_{1}$ as the norm in our computation. 